\title{
Synthesis of Variable Gain Robust Controllers for a Class of Uncertain Dynamical Systems
}

\author{
Hidetoshi Oya ${ }^{1}$ and Kojiro Hagino ${ }^{2}$ \\ ${ }^{1}$ The University of Tokushima \\ ${ }^{2}$ The University of Electro-Communications \\ Japan
}

\section{Introduction}

Robustness of control systems to uncertainties has always been the central issue in feedback control and therefore for dynamical systems with unknown parameters, a large number of robust controller design methods have been presented (e.g. (3; 37)). Also, many robust state feedback controllers achieving some robust performances such as quadratic cost function(28; 31), $\mathcal{H}^{\infty}$-disturbance attenuation(6) and so on have been suggested. It is well-known that most of these problems are reduced to standard convex optimization problems involving linear matrix inequalities (LMIs) which can be solved numerically very efficiently. Furthermore, in the case that the full state of systems cannot be measured, the control strategies via observer-based robust controllers (e.g. $(12 ; 19 ; 27)$ ) or robust output feedback one (e.g. $(9 ; 11)$ ) have also been well studied. However, most of the resulting controllers derived in the existing results have fixed structure, and these methods result in worst-case design. Therefore these controllers become cautious when the perturbation region of the uncertainties has been estimated larger than the proper region, because the robust controller designed by the existing results only has a fixed gain.

From these viewpoints, it is important to derive robust controllers with adjustable parameters which are tuned by using available information. Thus some researchers have proposed robust controllers with adjustable parameters $(18 ; 33)$. In the work of Ushida et al.(33), a quadratically stabilizing state feedback controller based on the parametrization of $\mathcal{H}^{\infty}$ controllers is derived. Maki and Hagino(18) have introduced a robust controller with adaptation mechanism for linear systems with time-varying parameter uncertainties and the controller gain in their work is tuned on-line based on the information about parameter uncertainties. On the other hand, we have proposed a robust controller with adaptive compensation input for a class of uncertain linear systems $(19 ; 21 ; 22)$. The adaptive compensation input is tuned by adjustable parameters based on the error information between the plant trajectory and the desired one. These adaptive robust controllers achieve good control performance and these approaches are very simple due to the application of the linear quadratic control problem. Besides these design methods reduce the cautiousness in a robust controller with a fixed gain, because utilizing the error signal between the real response of the uncertain system and the desired one is equivalent to giving consideration to the effect of the uncertainties as on-line information. 
In this chapter, for a class of uncertain linear systems, variable gain robust controllers which achieve not only asymptotical stability but also improving transient behavior of the resulting closed-loop system have been shown $(23 ; 24 ; 26)$. The variable gain robust controllers, which consist of fixed gain controllers and variable gain one, are tuned on-line based on the information about parameter uncertainties. In this chapter, firstly, a design method of variable gain state feedback controllers for linear systems with matched uncertainties has been shown and next the variable gain state feedback controller is extended to output feedback controllers. Finally, on the basis of the concept of piecewise Lyapunov functions (PLFs), an LMI-based variable gain robust controller synthesis for linear systems with matched uncertainties and unmatched one has been presented.

The contents of this chapter are as follows, where the item numbers in the list accord with the section numbers.

2. Variable Gain Robust State Feedback Controllers

3. Variable Gain Robust Output Feedback Controllers

4. Variable Gain Robust Controllers based on Piecewise Lyapunov Functions

5. Conclusions and Future Works

Basic symbols are listed bellow.

$$
\begin{array}{ll}
\mathbb{Z}^{+} & : \text {the set of positive integers } \\
\mathbb{R} & : \text { the set of real numbers } \\
\mathbb{R}^{n} & : \text { the set of } n \text {-dimensional vectors } \\
\mathbb{R}^{n \times m} & : \text { the set of } n \times m \text {-dimensional matrices } \\
I_{n} & : n \text {-dimensional identity matrix }
\end{array}
$$

Other than the above, we use the following notation and terms. For a matrix $\mathcal{A}$, the transpose of matrix $\mathcal{A}$ and the inverse of one are denoted by $\mathcal{A}^{T}$ and $\mathcal{A}^{-1}$ respectively and $\operatorname{rank}\{\mathcal{A}\}$ represents the rank of the matrix $\mathcal{A}$. Also, $I_{n}$ represents $n$-dimensional identity matrix. For real symmetric matrices $\mathcal{A}$ and $\mathcal{B}, \mathcal{A}>\mathcal{B}$ (resp. $\mathcal{A} \geq \mathcal{B}$ ) means that $\mathcal{A}-\mathcal{B}$ is positive (resp. nonnegative) definite matrix. For a vector $\alpha \in \mathbb{R}^{n},\|\alpha\|$ denotes standard Euclidian norm and for a matrix $\mathcal{A},\|\mathcal{A}\|$ represents its induced norm. Besides, a vector $\alpha \in \mathbb{R}^{n},\|\alpha\|_{1}$ denotes 1-norm, i.e. $\|\alpha\|_{1}$ is defined as $\|\alpha\|_{1} \triangleq \sum_{j=1}^{n}\left|\alpha_{j}\right|$. The intersection of two sets $\Gamma$ and $Y$ are denoted by $\Gamma \cap Y$ and the symbols " $\triangleq$ " and " $\star$ " mean equality by definition and symmetric blocks in matrix inequalities, respectively. Besides, for a symmetric matrix $\mathcal{P}, \lambda_{\max }\{\mathcal{P}\}$ (resp. $\lambda_{\min }\{\mathcal{P}\}$ ) represents the maximal eigenvalue (resp. minimal eigenvalue).

Furthermore, the following usefule lemmas are used in this paper.

Lemma 1. For arbitrary vectors $\lambda$ and $\xi$ and the matrices $\mathcal{G}$ and $\mathcal{H}$ which have appropriate dimensions, the following relation holds.

$$
\begin{aligned}
2 \lambda^{T} \mathcal{G} \Delta(t) \mathcal{H} \xi & \leq 2\left\|\mathcal{G}^{T} \lambda\right\|\|\Delta(t) \mathcal{H} \xi\| \\
& \leq 2\left\|\mathcal{G}^{T} \lambda\right\|\|\mathcal{H} \xi\|
\end{aligned}
$$

where $\Delta(t) \in \mathbb{R}^{p \times q}$ is a time-varying unknown matrix satisfying $\|\Delta(t)\| \leq 1$.

Proof. The above relation can be easily obtained by Schwartz's inequality (see (8)). 
Lemma 2. (Schur complement) For a given constant real symmetric matrix $\Xi$, the following arguments are equivalent.

(i). $\Xi=\left(\begin{array}{ll}\Xi_{11} & \Xi_{12} \\ \Xi_{12}^{T} & \Xi_{22}\end{array}\right)>0$

(ii). $\Xi_{11}>0$ and $\Xi_{22}-\Xi_{12}^{T} \Xi_{11}^{-1} \Xi_{12}>0$

(iii). $\Xi_{22}>0$ and $\Xi_{11}-\Xi_{12} \Xi_{22}^{-1} \Xi_{12}^{T}>0$

Proof. See Boyd et al.(4)

Lemma 3. ( Barbalat's lemma) Let $\phi: \mathbb{R} \rightarrow \mathbb{R}$ be a uniformly continuous function on $[0, \infty)$. Suppose that $\lim _{t \rightarrow \infty} \int_{0}^{t} \phi(\tau) d \tau$ exists and is finite. Then

$$
\phi(t) \rightarrow 0 \text { as } t \rightarrow \infty
$$

Proof. See Khalil(13).

Lemma 4. (S-procedure) Let $\mathcal{F}(x)$ and $\mathcal{G}(x)$ be two arbitrary quadratic forms over $\mathbb{R}^{n}$. Then $\mathcal{F}(x)<$ 0 for $\forall x \in \mathbb{R}^{n}$ satisfying $\mathcal{G}(x) \leq 0$ if and only if there exist a nonnegative scalar $\tau$ such that

$$
\mathcal{F}(x)-\tau \mathcal{G}(x) \leq 0 \text { for } \forall x \in \mathbb{R}^{n}
$$

Proof. See Boyd et al.(4).

\section{Variable gain robust state feedback controllers}

In this section, we propose a variable gain robust state feedback controller for a class of uncertain linear systems. The uncertainties under consideration are supposed to satisfy the matching condition(3) and the variable gain robust state feedback controller under consideration consists of a state feedback with a fixed gain matrix and a compensation input with variable one. In this section, we show a design method of the variable gain robust state feedback controller.

\subsection{Problem formulation}

Consider the uncertain linear system described by the following state equation.

$$
\frac{d}{d t} x(t)=(A+B \Delta(t) E) x(t)+B u(t)
$$

where $x(t) \in \mathbb{R}^{n}$ and $u(t) \in \mathbb{R}^{m}$ are the vectors of the state (assumed to be available for feedback) and the control input, respectively. In (2.1) the matrices $A$ and $B$ denote the nominal values of the system, and the pair $(A, B)$ is stabilizable and the matrix $\Delta(t) \in \mathbb{R}^{m \times q}$ denotes unknown time-varying parameters which satisfy $\|\Delta(t)\| \leq 1$. Namely, the uncertain parameter satisfies the matching condition (See e.g. (3) and references therein).

The nominal system, ignoring the unknown parameter $\Delta(t)$ in (2.1), is given by

$$
\frac{d}{d t} \bar{x}(t)=A \bar{x}(t)+B \bar{u}(t)
$$

where $\bar{x}(t) \in \mathbb{R}^{n}$ and $\bar{u}(t) \in \mathbb{R}^{m}$ are the vectors of the state and the control input for the nominal system respectively. 
First of all, in order to generate the desirable transient behavior in time response for the uncertain system (2.1) systematically, we adopt the standard linear quadratic control problem (LQ control theory) for the nominal system (2.2). Note that some other design method so as to generate the desired response for the controlled system can also be used (e.g. pole assignment). It is well-known that the optimal control input for the nominal system (2.2) can be obtained as $\bar{u}(t)=-K \bar{x}(t)$ and the closed-loop system

$$
\begin{aligned}
\frac{d}{d t} \bar{x}(t) & =(A+B K) \bar{x}(t) \\
& =A_{K} \bar{x}(t)
\end{aligned}
$$

is asymptotically stable*.

Now in order to obtain on-line information on the parameter uncertainty, we introduce an error signal $e(t) \triangleq x(t)-\bar{x}(t)$, and for the uncertain system (2.1), we consider the following control input.

$$
u(t) \triangleq K x(t)+\psi(x, e, \mathcal{L}, t)
$$

where $\psi(x, e, \mathcal{L}, t) \in \mathbb{R}^{m}$ is a compensation input(21) to correct the effect of uncertainties, and it is supposed to have the following structure.

$$
\psi(x, e, \mathcal{L}, t) \triangleq \mathcal{F} e(t)+\mathcal{L}(x, e, t) e(t)
$$

In (2.4), $\mathcal{F} \in \mathbb{R}^{\mathbb{R}^{m \times n}}$ and $\mathcal{L}(t) \in \mathbb{R}^{m \times n}$ are a fixed gain matrix and an adjustable time-varying matrix, respectively. Thus from (2.1), (2.3) - (2.5), the error system can be written as

$$
\begin{aligned}
\frac{d}{d t} e(t) & =(A+B \Delta(t) E) x(t)+B(K x(t)+\mathcal{F} e(t)+\mathcal{L}(x, e, t) x(t))-A_{K} \bar{x}(t) \\
& =A_{\mathcal{F}} e(t)+B \Delta(t) E x(t)+\mathcal{L}(x, e, t) e(t)
\end{aligned}
$$

In (2.6), $A_{\mathcal{F}}$ is the matrix expressed as

$$
A_{\mathcal{F}}=A_{K}+B \mathcal{F}
$$

Note that from the definition of the error signal, the uncertain system (2.1) is ensured to be stable, because the nominal system is asymptotically stable.

From the above, our control objective in this section is to derive the fixed gain matrix $\mathcal{F} \in \mathbb{R}^{m \times n}$ and the variable gain matrix $\mathcal{L}(x, e, t) \in \mathbb{R}^{m \times n}$ which stabilize the uncertain error system (2.6).

\footnotetext{
${ }^{*}$ Using the unique solution of the algebraic Riccati equation $A^{T} \mathcal{X}+\mathcal{X} A-\mathcal{X} B \mathcal{R}^{-1} B^{T} \mathcal{X}+\mathcal{Q}=0$, the gain matrix $K \in \mathbb{R}^{m \times n}$ is determined as $K=-\mathcal{R}^{-1} B^{T} \mathcal{X}$ where $\mathcal{Q}$ and $\mathcal{R}$ are nonnegative and positive definite matrices, respectively. Besides, $\mathcal{Q}$ is selected such that the pair $(A, \mathcal{C})$ is detectable, where $\mathcal{C}$ is any matrix satisfying $\mathcal{Q}=\mathcal{C C}^{T}$, and then the matrix $A_{K} \triangleq A+B K$ is stable.
} 


\subsection{Synthesis of variable gain robust state feedback controllers}

In this subsection, we consider designing the variable matrix $\mathcal{L}(x, e, t) \in \mathbb{R}^{m \times n}$ and the fixed gain matrix $\mathcal{F} \in \mathbb{R}^{m \times n}$ such that the error system (2.6) with unknown parameters is asymptotically stable. The following theorem gives a design method of the proposed adaptive robust controller.

Theorem 1. Consider the uncertain error system (2.6) with variable gain matrix $\mathcal{L}(x, e, t) \in \mathbb{R}^{m \times n}$ and the fixed gain matrix $\mathcal{F} \in \mathbb{R}^{m \times n}$.

By using the $L Q$ control theory, the fixed gain matrix $\mathcal{F} \in \mathbb{R}^{m \times n}$ is designed as $\mathcal{F}=-\mathcal{R}_{e}^{-1} B^{T} \mathcal{P}$ where $\mathcal{P} \in \mathbb{R}^{n \times n}$ is unique solution of the following algebraic Riccati equation.

$$
A_{K}^{T} \mathcal{P}+\mathcal{P} A_{K}-\mathcal{P} B \mathcal{R}_{e}^{-1} B^{T} \mathcal{P}+\mathcal{Q}_{e}=0
$$

where $\mathcal{Q}_{e} \in \mathbb{R}^{n \times n}$ and $\mathcal{R}_{e} \in \mathbb{R}^{m \times m}$ are positive definite matrices which are selected by designers. Besides, the variable gain matrix $\mathcal{L}(x, e, t) \in \mathbb{R}^{m \times n}$ is determined as

$$
\mathcal{L}(x, e, t)=-\frac{\|E x(t)\|^{2}}{\left\|B^{T} \mathcal{P} e(t)\right\|\|E x(t)\|+\sigma(t)} B^{T} \mathcal{P}
$$

In (2.9), $\sigma(t) \in \mathbb{R}^{+}$is any positive uniform continuous and bounded function which satisfies

$$
\int_{t_{0}}^{t} \sigma(\tau) d \tau \leq \sigma^{*}<\infty
$$

where $\sigma^{*}$ is any positive constant and $t_{0}$ denotes an initial time. Then the uncertain error system (2.6) is bounded and

$$
\lim _{t \rightarrow \infty} e\left(t ; t_{0}, e\left(t_{0}\right)\right)=0
$$

Namely, asymptotical stability of the uncertain error system (2.6) is ensured.

Proof. Using symmetric positive definite matrix $\mathcal{P} \in \mathbb{R}^{n \times n}$ which satisfies (2.8), we introduce the following quadratic function

$$
\mathcal{V}(e, t) \triangleq e^{T}(t) \mathcal{P} e(t)
$$

Let's $e(t)$ be the solution of (2.6) for $t \geq t_{0}$. Then the time derivative of the function $\mathcal{V}(e, t)$ along the trajectory of (2.6) can be written as

$$
\begin{aligned}
\frac{d}{d t} \mathcal{V}(e, t)= & e^{T}(t)\left(A_{\mathcal{F}}^{T} \mathcal{P}+\mathcal{P} A_{\mathcal{F}}\right) e(t) \\
& +2 e^{T}(t) \mathcal{P} B \Delta(t) E x(t)+2 e^{T}(t) \mathcal{P} B \mathcal{L}(x, e, t) e(t)
\end{aligned}
$$

Now, one can see from (2.13) and Lemma 1 that the following inequality for the function $\mathcal{V}(e, t)$ holds.

$$
\begin{aligned}
\frac{d}{d t} \mathcal{V}(e, t)= & e^{T}(t)\left(A_{\mathcal{F}}^{T} \mathcal{P}+\mathcal{P} A_{\mathcal{F}}\right) e(t)+2\left\|B^{T} \mathcal{P} e(t)\right\|\|\Delta(t) E x(t)\| \\
& +2 e^{T}(t) \mathcal{P} B \mathcal{L}(x, e, t) e(t) \\
\leq & e^{T}(t)\left(A_{\mathcal{F}}^{T} \mathcal{P}+\mathcal{P} A_{\mathcal{F}}\right) e(t)+2\left\|B^{T} \mathcal{P} e(t)\right\|\|E x(t)\| \\
& +2 e^{T}(t) P B \mathcal{L}(x, e, t) e(t)
\end{aligned}
$$


Additionally, using the relation (2.8), substituting (2.9) into (2.14) and some trivial manipulations give the inequality

$$
\begin{aligned}
\frac{d}{d t} \mathcal{V}(e, t) \leq & e^{T}(t)\left(A_{\mathcal{F}}^{T} \mathcal{P}+\mathcal{P} A_{\mathcal{F}}\right) e(t)+2\left\|B^{T} \mathcal{P} e(t)\right\|\|E x(t)\| \\
& +2 e^{T}(t) \mathcal{P} B\left(-\frac{\|E x(t)\|^{2}}{\left\|B^{T} \mathcal{P} e(t)\right\|\|E x(t)\|+\sigma(t)} B^{T} \mathcal{P}\right) e(t) \\
\leq & e^{T}(t)\left(A_{\mathcal{F}}^{T} \mathcal{P}+\mathcal{P} A_{\mathcal{F}}\right) e(t)+2 \frac{\left\|B^{T} \mathcal{P} e(t)\right\|\|E x(t)\|}{\left\|B^{T} \mathcal{P} e(t)\right\|\|E x(t)\|+\sigma(t)} \sigma(t) \\
= & -e^{T}(t)\left\{\mathcal{Q}_{e}+\mathcal{P} B \mathcal{R}_{e}^{-1} B^{T} \mathcal{P}\right\} e(t)+2 \frac{\left\|B^{T} \mathcal{P} e(t)\right\|\|E x(t)\|}{\left\|B^{T} \mathcal{P} e(t)\right\|\|E x(t)\|+\sigma(t)} \sigma(t)
\end{aligned}
$$

Notice the fact that for $\forall \alpha, \beta>0$

$$
0 \leq \frac{\alpha \beta}{\alpha+\beta} \leq \alpha
$$

Then we can further obtain that for any $t>t_{0}$

$$
\frac{d}{d t} \mathcal{V}(e, t) \leq-e(t) \Omega e(t)+\sigma(t)
$$

where $\Omega \in \mathbb{R}^{n \times n}$ is the symmetric positive definite matrix given by

$$
\Omega=\mathcal{Q}_{e}+\mathcal{P} B \mathcal{R}_{e}^{-1} B^{T} \mathcal{P}
$$

Besides, letting $\zeta \triangleq \lambda_{\min }(\Omega)$, we have

$$
\frac{d}{d t} \mathcal{V}(e, t) \leq-\zeta\|e(t)\|^{2}+\sigma(t)
$$

On the other hand, from the definition of the quadratic function $\mathcal{V}(e, t)$, there always exist two positive constants $\xi^{-}$and $\xi^{+}$such that for any $t \geq t_{0}$,

$$
\xi^{-}(\|e(t)\|) \leq \mathcal{V}(e, t) \leq \xi^{+}(\|e(t)\|)
$$

where $\xi^{-}(\|e(t)\|)$ and $\xi^{+}(\|e(t)\|)$ are given by

$$
\begin{aligned}
& \xi^{-}(\|e(t)\|) \triangleq \xi^{-}\|e(t)\|^{2} \\
& \xi^{+}(\|e(t)\|) \triangleq \xi^{+}\|e(t)\|^{2}
\end{aligned}
$$

From the above, we want to show that the solution $e(t)$ is uniformly bounded, and that the error signal $e(t)$ converges asymptotically to zero.

By continuity of the error system (2.6), it is obvious that any solution $e\left(t ; t_{0}, e\left(t_{0}\right)\right)$ of the error system is continuous. Namely, $e(t)$ is also continuous, because the state $\bar{x}(t)$ for the nominal 
system is continuous. In addition, it follows from (2.19) and (2.20), that for any $t \geq t_{0}$, we have

$$
\begin{aligned}
& 0 \leq \xi^{-}(\|e(t)\|) \leq \mathcal{V}(e, t)=\mathcal{V}\left(e, t_{0}\right)+\int_{t_{0}}^{t} \frac{d}{d t} \mathcal{V}(e, \tau) d \tau \\
& \mathcal{V}(e, t) \leq \xi^{+}\left(\left\|e\left(t_{0}\right)\right\|\right)-\int_{t_{0}}^{t} \xi^{*}(\|e(\tau)\|) d \tau+\int_{t_{0}}^{t} \sigma(\tau) d \tau
\end{aligned}
$$

In (2.23), $\xi^{*}(\|e(t)\|)$ is defined as

$$
\xi^{*}(\|e(t)\|) \triangleq \zeta\|e(t)\|^{2}
$$

Therefore, from (2.22) and (2.23) we can obtain the following two results. Firstly, taking the limit as $t$ approaches infinity on both sides of inequality (2.23), we have the following relation.

$$
0 \leq \xi^{+}\left(\left\|e\left(t_{0}\right)\right\|\right)-\lim _{t \rightarrow \infty} \int_{t_{0}}^{t} \xi^{*}(\|e(\tau)\|) d \tau+\lim _{t \rightarrow \infty} \int_{t_{0}}^{t} \sigma(\tau) d \tau
$$

Thus one can see from (2.10) and (2.25) that

$$
\lim _{t \rightarrow \infty} \int_{t_{0}}^{t} \xi^{*}(\|e(\tau)\|) d \tau \leq \xi^{+}\left(\left\|e\left(t_{0}\right)\right\|\right)+\sigma^{*}
$$

On the other hand, from (2.22) and (2.23), we obtain

$$
0 \leq \xi^{-}(\|e(t)\|) \leq \xi^{+}\left(\left\|e\left(t_{0}\right)\right\|\right)+\int_{t_{0}}^{t} \sigma(\tau) d \tau
$$

Note that for any $t \geq t_{0}$,

$$
\sup _{t \in\left[t_{0}, \infty\right)} \int_{t_{0}}^{t} \sigma(\tau) d \tau \leq \sigma^{*}
$$

It follows from (2.27) and (2.28) that

$$
0 \leq \xi^{-}(\|e(t)\|) \leq \xi^{+}\left(\left\|e\left(t_{0}\right)\right\|\right)+\sigma^{*}
$$

The relation (2.29) implies that $e(t)$ is uniformly bounded. Since $e(t)$ has been shown to be continuous, it follows that $e(t)$ is uniformly continuous. Therefore that $e(t)$ is uniformly continuous and one can see from the definition that the function $\xi^{*}(\|e(t)\|)$ also uniformly continuous. Applying the Lemma 2 (Barbalat's lemma) to (2.26) yields

$$
\lim _{t \rightarrow \infty} \xi^{*}(\|e(t)\|)=0
$$

Besides, since $\xi^{*}(\|e(t)\|)$ is a positive definite scalar function, it is obvious that the following equation holds.

$$
\lim _{t \rightarrow \infty}\|e(t)\|=0
$$

Namely, asymptotical stability of the uncertain error system (2.6) is ensured. Therefore the uncertain system (2.1) is also asymptotically stable, because the nominal system (2.2) is stable. It follows that the result of the theorem is true. Thus the proof of Theorem $\mathbf{1}$ is completed. 
Remark 1. Though, the variable gain controllers in the existing results $19 ; 21)$ can also be good transient performance, these controllers may cause serious chattering, because the adjustment parameters in the existing results $(19 ; 21)$ are adjusted on the boundary surface of the allowable parameter space (see. (26) for details). On the other hand, since the variable gain matrix (2.9) of the proposed robust controller is continuous, chattering phenomenon can be avoided.

\subsection{Illustrative examples}

In order to demonstrate the efficiency of the proposed control scheme, we have run a simple example.

Consider the following linear system with unknown parameter, i.e. the unknown parameter $\Delta(t) \in \mathbb{R}^{1}$.

$$
\frac{d}{d t} x(t)=\left(\begin{array}{rr}
1 & 1 \\
0 & -2
\end{array}\right) x(t)+\left(\begin{array}{l}
0 \\
1
\end{array}\right) \Delta(t)\left(\begin{array}{ll}
5 & 4
\end{array}\right)+\left(\begin{array}{l}
0 \\
1
\end{array}\right) u(t)
$$

Now we select the weighting matrices $\mathcal{Q}$ and $\mathcal{R}$ such as $\mathcal{Q}=1.0 I_{2}$ and $\mathcal{R}=4.0$ for the standard linear quadratic control problem for the nominal system, respectively. Then solving the algebraic Riccati equation, we obtain the following optimal gain matrix

$$
K=(-6.20233-2.08101)
$$

In addition, setting the design parameters $\mathcal{Q}_{e}$ and $\mathcal{R}_{e}$ such as $\mathcal{Q}_{e}=9.0 I_{2}$ and $\mathcal{R}_{e}=1.0$, respectively, we have

$$
\mathcal{F}=\left(-2.37665 \times 10^{2}-9.83494 \times 10^{1}\right)
$$

Besides for the variable gain matrix $\mathcal{L}(x, e, t) \in \mathbb{R}^{m \times n}$, we select the following parameter.

$$
\sigma(t)=50 \exp (-0.75 t)
$$

In this example, we consider the following two cases for the unknown parameter $\Delta(t)$.

- Case 1) :

$$
\Delta(t)=\sin (\pi t)
$$

- Case 2) :

$$
\begin{aligned}
& \Delta(t)=-1.0: 0 \leq t \leq 1.0 \\
& \Delta(t)=1.0 \quad: 1.0<t \leq 2.0 \\
& \Delta(t)=-1.0: t>2.0
\end{aligned}
$$

Besides, for numerical simulations, the initial values of the uncertain system (2.32) and the nominal system are selected as $x(0)=\bar{x}(0)=\left(\begin{array}{ll}1.0 & -1.0\end{array}\right)^{T}$.

\begin{tabular}{|c||c||c|}
\hline & $\mathcal{J}^{1}(e, t)$ & $\mathcal{J}^{2}(e, t)$ \\
\hline \hline Case 1) & $1.05685 \times 10^{-4}$ & $1.41469 \times 10^{-3}$ \\
\hline \hline Case 2) & $2.11708 \times 10^{-4}$ & $2.79415 \times 10^{-3}$ \\
\hline
\end{tabular}

Table 1. The values of the performance indecies 


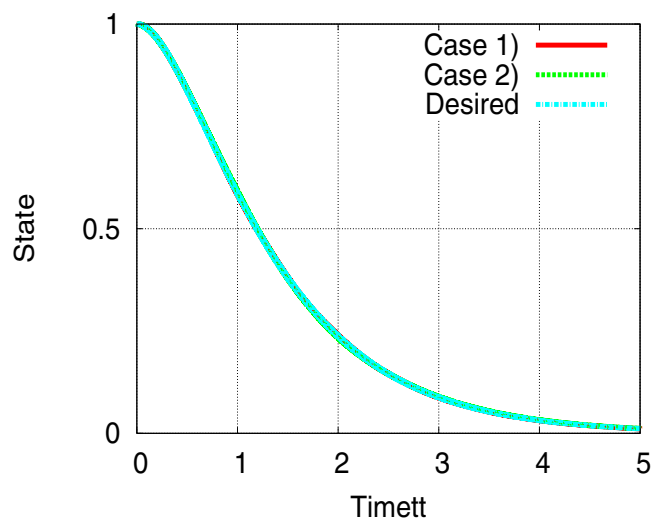

Fig. 1. Time histories of the state $x_{1}(t)$

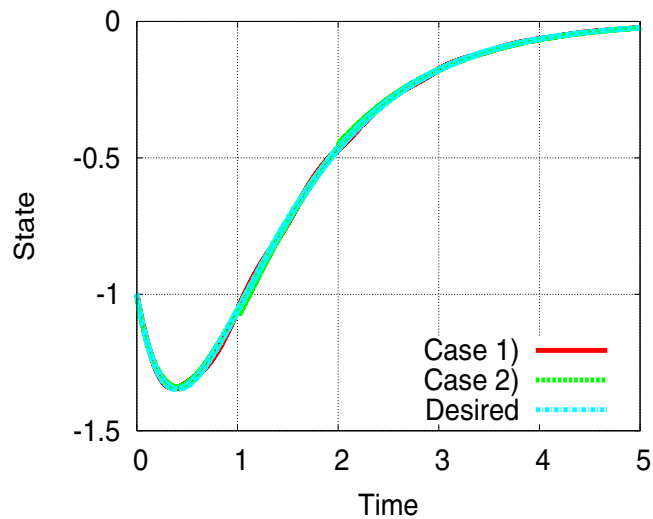

Fig. 2. Time histories of the state $x_{2}(t)$

The results of the simulation of this example are depicted in Figures 1-3 and Table 1. In these Figures, "Case 1)" and "Case 2)" represent the time-histories of the state variables $x_{1}(t)$ and $x_{2}(t)$ and the control input $u(t)$ generated by the proposed controller, and "Desired" shows the desired time-response and the desired control input generated by the nominal system. Additionally $\mathcal{J}^{k}(e, t)(k=1,2)$ in Table 1 represent the following performance indecies.

$$
\begin{aligned}
& \mathcal{J}^{1}(e, t) \triangleq \int_{0}^{\infty} e^{T}(t) e(t) d t \\
& \mathcal{J}^{2}(e, t) \triangleq \sup _{t}\|e(t)\|_{1}
\end{aligned}
$$

From Figures 1-3, we find that the proposed variable gain robust state feedback controller stabilizes the uncertain system (2.32) in spite of uncertainties. Besides one can also see from Figures 1 and 2 and Table 1 that the proposed variable gain robust state feedback controller achieves the good transient performance and can avoid serious chattering. 


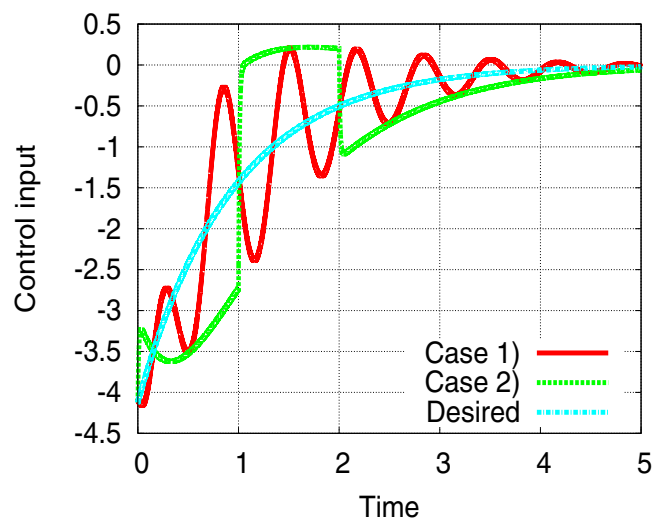

Fig. 3. Time histories of the control input $u(t)$

\subsection{Summary}

In this section, a design method of a variable gain robust state feedback controller for a class of uncertain linear systems has been presented and, by numerical simulations, the effectiveness of the proposed controller has been presented.

Since the proposed state feedback controller can easily be obtained by solving the standard algebraic Riccati equation, the proposed design approach is very simple. The proposed variable gain robust state feedback controller can be extended to robust servo systems and robust tracking control systems.

\section{Variable gain robust output feedback controllers}

In section 2, it is assumed that all the state are measurable and the procedure specifies the current control input as a function of the current value of the state vector. However it is physically and economically impractical to measure all of the state in many practical control systems. Therefore, it is necessary that the control input from the measurable signal is constructed to achieve satisfactory control performance. In this section, for a class of uncertain linear systems, we extend the result derived in section 2 to a variable gain robust output feedback controller.

\subsection{Problem formulation}

Consider the uncertain linear system described by the following state equation.

$$
\begin{aligned}
\frac{d}{d t} x(t) & =(A+B \Delta(t) E) x(t)+B u(t) \\
y(t) & =C x(t)
\end{aligned}
$$

where $x(t) \in \mathbb{R}^{n}, u(t) \in \mathbb{R}^{m}$ and $y(t) \in \mathbb{R}^{l}$ are the vectors of the state, the control input and the measured output, respectively. In (3.1), the matrices $A, B$ and $C$ are the nominal values of system parameters and the matrix $\Delta(t) \in \mathbb{R}^{p \times q}$ denotes unknown time-varying parameters which satisfy $\|\Delta(t)\| \leq 1$. In this paper, we introduce the following assumption for the system parameters(25).

$$
B^{T}=\mathcal{T} C
$$


where $\mathcal{T} \in \mathbb{R}^{m \times l}$ is a known constant matrix.

The nominal system, ignoring unknown parameters in (3.1), is given by

$$
\begin{aligned}
\frac{d}{d t} \bar{x}(t) & =A \bar{x}(t)+B \bar{u}(t) \\
\bar{y}(t) & =C \bar{x}(t)
\end{aligned}
$$

In this paper, the nominal system (3.3) is supposed to be stabilizable via static output feedback control. Namely, there exists an output feedback control $\bar{u}(t)=K \bar{y}(t)$ (i.e. a fixed gain matrix $\left.K \in \mathbb{R}^{m \times l}\right)$. In other words, since the nominal system is stabilizable via static output feedback control, the matrix $A_{K} \triangleq A+B K C$ is asymptotically stable. Note that the feedback gain matrix $K \in \mathbb{R}^{m \times l}$ is designed by using the existing results (e.g. $(2 ; 16)$ ).

Now on the basis of the work of (25), we introduce the error vectors $e(t) \triangleq x(t)-\bar{x}(t)$ and $e_{y}(t) \triangleq y(t)-\bar{y}(t)$. Beside, using the fixed gain matrix $K \in \mathbb{R}^{m \times l}$, we consider the following control input for the uncertain linear system (3.1).

$$
u(t) \triangleq K y(t)+\psi\left(e_{y}, \mathcal{L}, t\right)
$$

where $\psi\left(e_{y}, \mathcal{L}, t\right) \in \mathbb{R}^{m}$ is a compensation input (e.g. (25)) and has the following form.

$$
\psi\left(e_{y}, \mathcal{L}, t\right) \triangleq \mathcal{L}\left(e_{y}, t\right) e_{y}(t)
$$

In (3.5), $\mathcal{L}\left(e_{y}, t\right) \in \mathbb{R}^{m \times l}$ is a variable gain matrix. Then one can see from (3.1) and (3.3) - (3.5) that the following uncertain error system can be derived.

$$
\begin{aligned}
\frac{d}{d t} e(t) & =A_{K} e(t)+B \Delta(t) E x(t)+B \mathcal{L}\left(e_{y}, t\right) e_{y}(t) \\
e_{y}(t) & =C e(t)
\end{aligned}
$$

From the above, our control objective is to design the variable gain robust output feedback controller which stabilizes the uncertain error system (3.6). That is to derive the variable gain matrix $\mathcal{L}\left(e_{y}, t\right) \in \mathbb{R}^{m \times l}$ which stabilizes the uncertain error system (3.6).

\subsection{Synthesis of variable gain robust output feedback controllers}

In this subsection, an LMI-based design method of the variable gain robust output feedback controller for the uncertain linear system (3.1) is presented. The following theorem gives an LMI-based design method of a variable gain robust output feedback controller.

Theorem 2. Consider the uncertain error system (3.6) with the variable gain matrix $\mathcal{L}\left(e_{y}, t\right) \in \mathbb{R}^{m \times l}$. Suppose there exist the positive definite matrices $\mathcal{S} \in \mathbb{R}^{n \times n}, \Theta \in \mathbb{R}^{l \times l}$ and $\Psi \in \mathbb{R}^{l \times l}$ and the positive constants $\gamma_{1}$ and $\gamma_{2}$ satisfying the following LMIs.

$$
\begin{aligned}
& \mathcal{S} A_{K}+A_{K}^{T} \mathcal{S}+\gamma_{1} E^{T} E \leq-\mathcal{Q} \\
& -C^{T} \Theta C+\mathcal{S} C^{T} \mathcal{T}^{T} \mathcal{T} C+C \mathcal{T}^{T} \mathcal{T} C \mathcal{S} \leq 0 \\
& \left(\begin{array}{ccc}
-C^{T} \Psi C & \mathcal{S} C^{T} \mathcal{T} & \mathcal{S} C^{T} \mathcal{T} \\
\star & -\gamma_{1} I_{m} & 0 \\
\star & \star & -\gamma_{2} I_{m}
\end{array}\right) \leq 0
\end{aligned}
$$


Using the positive definite matrices $\Theta \in \mathbb{R}^{l \times l}$ and $\Psi \in \mathbb{R}^{l \times l}$, we consider the following variable gain matrix.

$$
\mathcal{L}\left(e_{y}, t\right)=-\frac{\left(\left\|\Psi^{1 / 2} C e(t)\right\|^{2}+\gamma_{2}\|E \bar{x}(t)\|^{2}\right)^{2}}{\left\|\Theta^{1 / 2} C e(t)\right\|^{2}\left(\left\|\Psi^{1 / 2} C e(t)\right\|^{2}+\gamma_{2}\|E \bar{x}(t)\|^{2}+\sigma(t)\right)} \mathcal{T}
$$

In (3.7), $\mathcal{Q} \in \mathbb{R}^{n \times n}$ is a symmetric positive definite matrix selected by designers and $\sigma(t) \in \mathbb{R}^{1}$ in (3.8) is any positive uniform continuous and bounded function which satisfies

$$
\int_{t_{0}}^{t} \sigma(\tau) d \tau \leq \sigma^{*}<\infty
$$

where $t_{0}$ and $\sigma^{*}$ are an initial time and any positive constant, respectively.

Then asymptotical stability of the uncertain error system (3.6) is guaranteed.

Proof. Firstly, we introduce the quadratic function $\mathcal{V}(e, t) \triangleq e^{T}(t) \mathcal{S} e(t)$. The time derivative of the quadratic function $\mathcal{V}(e, t)$ can be written as

$$
\frac{d}{d t} \mathcal{V}(e, t)=e^{T}(t)\left(\mathcal{S} A_{K}+A_{K}^{T} \mathcal{S}\right) e(t)+2 e^{T}(t) \mathcal{S} B \Delta(t) E x(t)+2 e^{T}(t) \mathcal{S} B \mathcal{L}\left(e_{y}, t\right) e_{y}(t)
$$

Now, using Lemma 1 and the assumption (3.2) we can obtain

$$
\begin{aligned}
\frac{d}{d t} \mathcal{V}(e, t) \leq & e^{T}(t)\left(\mathcal{S} A_{K}+A_{K}^{T} \mathcal{S}\right) e(t)+2 e^{T}(t) \mathcal{S} B \Delta(t) E(e(t)+\bar{x}(t))+2 e^{T}(t) \mathcal{S} B \mathcal{L}\left(e_{y}, t\right) e_{y}(t) \\
\leq & e^{T}(t)\left(\mathcal{S} A_{K}+A_{K}^{T} \mathcal{S}+\gamma_{1} E^{T} E\right) e(t)+2 e^{T}(t) \mathcal{S} C^{T} \mathcal{T}^{T} \mathcal{L}\left(e_{y}, t\right) e_{y}(t) \\
& +\frac{1}{\gamma_{1}} e^{T}(t) \mathcal{S} C^{T} \mathcal{T}^{T} \mathcal{T} C \mathcal{S} e(t)+\frac{1}{\gamma_{2}} e^{T}(t) \mathcal{S} C^{T} \mathcal{T}^{T} \mathcal{T} C \mathcal{S} e(t)+\gamma_{2} \bar{x}^{T}(t) E^{T} E \bar{x}(t)
\end{aligned}
$$

Here we have used the well-known following relation.

$$
2 a^{T} b \leq \mu a^{T} a+\frac{1}{\mu} b^{T} b
$$

where $a$ and $b$ are any vectors with appropriate dimensions and $\mu$ is any positive constant. Besides, we have the following inequality for the time derivative of the quadratic function $\mathcal{V}(e, t)$.

$$
\begin{aligned}
\frac{d}{d t} \mathcal{V}(e, t) \leq & e^{T}(t)\left(\mathcal{S} A_{K}+A_{K}^{T} \mathcal{S}+\gamma_{1} E^{T} E\right) e(t)+e^{T}(t) C^{T} \Psi C e(t)+\gamma_{2} \bar{x}^{T}(t) E^{T} E \bar{x}(t) \\
& +2 e^{T}(t) \mathcal{S} C^{T} \mathcal{T}^{T} \mathcal{L}\left(e_{y}, t\right) e_{y}(t)
\end{aligned}
$$

because by using Lemma 2 (Schur complement) the third LMI of (3.7) can be written as

$$
-C^{T} \Psi C+\frac{1}{\gamma_{1}} \mathcal{S} C^{T} \mathcal{T}^{T} \mathcal{T} C \mathcal{S}+\frac{1}{\gamma_{2}} \mathcal{S} C^{T} \mathcal{T}^{T} \mathcal{T} C \mathcal{S} \leq 0
$$


Furthermore using the variable gain matrix (3.8), the LMIs (3.7) and the well-known inequality for any positive constants $\alpha$ and $\beta$

$$
0 \leq \frac{\alpha \beta}{\alpha+\beta} \leq \alpha \quad \forall \alpha, \beta>0
$$

and some trivial manipulations give the following relation.

$$
\frac{d}{d t} \mathcal{V}(e, t) \leq-e^{T}(t) \mathcal{Q} e(t)+\sigma(t)
$$

In addition, by letting $\zeta \triangleq \min \left\{\lambda_{\min }\{\mathcal{Q}\}\right\}$, we obtain the following inequality.

$$
\frac{d}{d t} \mathcal{V}(e, t) \leq-\zeta\|e(t)\|^{2}+\sigma(t)
$$

On the other hand, one can see from the definition of the quadratic function $\mathcal{V}(e, t)$ that there always exist two positive constants $\delta_{\min }$ and $\delta_{\max }$ such that for any $t \geq t_{0}$,

$$
\xi^{-}(\|e(t)\|) \leq \mathcal{V}(e, t) \leq \xi^{+}(\|e(t)\|)
$$

where $\xi^{-}(\|e(t)\|)$ and $\xi^{+}(\|e(t)\|)$ are given by

$$
\begin{aligned}
& \xi^{-}(\|e(t)\|) \triangleq \delta_{\text {min }}\|e(t)\|^{2} \\
& \xi^{+}(\|e(t)\|) \triangleq \delta_{\text {max }}\|e(t)\|^{2}
\end{aligned}
$$

It is obvious that any solution $e\left(t ; t_{0}, e\left(t_{0}\right)\right)$ of the uncertain error system (3.6) is continuous. In addition, it follows from (3.17) and (3.18), that for any $t \geq t_{0}$, we have

$$
\begin{aligned}
& 0 \leq \xi^{-}(\|e(t)\|) \leq \mathcal{V}(e, t)=\mathcal{V}\left(e, t_{0}\right)+\int_{t_{0}}^{t} \frac{d}{d t} \mathcal{V}(e, \tau) d \tau \\
& \mathcal{V}\left(e, t_{0}\right)+\int_{t_{0}}^{t} \frac{d}{d t} \mathcal{V}(e, \tau) d \tau \leq \xi^{+}\left(\left\|e\left(t_{0}\right)\right\|\right)-\int_{t_{0}}^{t} \zeta(\|e(\tau)\|) d \tau+\int_{t_{0}}^{t} \sigma(\tau) d \tau
\end{aligned}
$$

In (3.20), $\xi^{*}(\|e(t)\|)$ is defined as

$$
\xi^{*}(\|e(t)\|) \triangleq \zeta\|e(t)\|^{2}
$$

Therefore, from (3.20) we can obtain the following two results. Firstly, taking the limit as $t$ approaches infinity on both sides of the inequality (3.20), we have

$$
0 \leq \xi^{+}\left(\left\|e\left(t_{0}\right)\right\|\right)-\lim _{t \rightarrow \infty} \int_{t_{0}}^{t} \xi^{*}(\|e(\tau)\|) d \tau+\lim _{t \rightarrow \infty} \int_{t_{0}}^{t} \sigma(\tau) d \tau
$$

Thus one can see from (3.9) and (3.22) that

$$
\lim _{t \rightarrow \infty} \int_{t_{0}}^{t} \xi^{*}(\|e(\tau)\|) d \tau \leq \xi^{+}\left(\left\|e\left(t_{0}\right)\right\|\right)+\sigma^{*}
$$

On the other hand, from (3.20), we obtain

$$
0 \leq \xi^{-}(\|e(t)\|) \leq \xi^{+}\left(\left\|e\left(t_{0}\right)\right\|\right)+\int_{t_{0}}^{t} \sigma(\tau) d \tau
$$


It follows from (3.9) and (3.24) that

$$
0 \leq \xi^{-}(\|e(t)\|) \leq \xi^{+}\left(\left\|e\left(t_{0}\right)\right\|\right)+\sigma^{*}
$$

The relation (3.25) implies that $e(t)$ is uniformly bounded. Since $e(t)$ has been shown to be continuous, it follows that $e(t)$ is uniformly continuous. Therefore, one can see from the definition that $\xi^{*}(\|e(t)\|)$ is also uniformly continuous. Thus applying Lemma 3 (Barbalat's lemma) to (3.23) yields

$$
\lim _{t \rightarrow \infty} \xi^{*}(\|e(t)\|)=\lim _{t \rightarrow \infty} \zeta\|e(t)\|^{2}=0
$$

Namely, asymptotical stability of the uncertain error system (3.6) is ensured. Thus the uncertain linear system (3.1) is also stable.

It follows that the result of the theorem is true. Thus the proof of Theorem $\mathbf{2}$ is completed.

Theorem 2 provides a sufficient condition for the existence of a variable gain robust output feedback controller for the uncertain linear system (3.1). Next, we consider a special case. In this case, we consider the uncertain linear system described by

$$
\begin{aligned}
\frac{d}{d t} x(t) & =(A+B \Delta(t) C) x(t)+B u(t) \\
y(t) & =C x(t)
\end{aligned}
$$

Thus one can see from (3.3) - (3.5) and (3.27) that we have the following uncertain error system.

$$
\begin{aligned}
\frac{d}{d t} e(t) & =A_{K} e(t)+B \Delta(t) C x(t)+B \mathcal{L}\left(e_{y}, t\right) e_{y}(t) \\
e_{y}(t) & =C e(t)
\end{aligned}
$$

Next theorem gives an LMI-based design method of a variable gain robust output feedback controller for this case.

Theorem 3. Consider the uncertain error system (3.28) with the variable gain matrix $\mathcal{L}\left(e_{y}, t\right) \in$ $\mathbb{R}^{m \times l}$.

Suppose there exist the symmetric positive definite matrices $\mathcal{X}>0, \mathcal{Y}>0$ and matrices $\mathcal{S} \in$ $\mathbb{R}^{n \times n}, \Theta \in \mathbb{R}^{l \times l}$ and $\Psi \in \mathbb{R}^{l \times l}$ and the positive constant $\gamma$ satisfying the LMIs.

$$
\begin{aligned}
& \mathcal{S} A_{K}+A_{K}^{T} \mathcal{S} \leq-\mathcal{Q} \quad\left(\mathcal{Q}=\mathcal{Q}^{T}>0\right) \\
& -C^{T} \Theta C+\mathcal{S} C^{T} \mathcal{T}^{T} \mathcal{T} C+C^{T} \mathcal{T}^{T} \mathcal{T} C \mathcal{S} \leq 0 \\
& \left(\begin{array}{cc}
-C^{T} \Psi C & \mathcal{S} C^{T} \mathcal{T} \\
\star & -\gamma I_{m}
\end{array}\right) \leq 0
\end{aligned}
$$

Using positive definite matrices $\Psi \in \mathbb{R}^{l \times l}$ and $\Theta \in \mathbb{R}^{l \times l}$ and the positive scalars $\gamma$ satisfying the LMIs (3.29), we consider the variable gain matrix

$$
\mathcal{L}\left(e_{y}, t\right)=-\frac{\left(\left\|\Psi^{1 / 2} e_{y}(t)\right\|^{2}+\gamma\|y(t)\|^{2}\right)^{2}}{\left\|\Theta^{1 / 2} C e(t)\right\|^{2}\left(\left\|\Psi^{1 / 2} e_{y}(t)\right\|^{2}+\gamma\|y(t)\|^{2}+\sigma(t)\right)} \mathcal{T}
$$

where $\sigma(t) \in \mathbb{R}^{1}$ is any positive uniform continuous and bounded function satisfying (3.9).

Then asymptotical stability of the uncertain error system (3.28) is guaranteed. 
Proof. By using the symmetric positive definite matrix $\mathcal{S} \in \mathbb{R}^{n \times n}$, we consider the quadratic function $\mathcal{V}(e, t) \triangleq e^{T}(t) \mathcal{S} e(t)$. Then using the assumption (3.2) we have

$$
\begin{aligned}
\frac{d}{d t} \mathcal{V}(e, t)= & e^{T}(t)\left(\mathcal{S} A_{K}+A_{K}^{T} \mathcal{S}\right) e(t)+2 e^{T}(t) \mathcal{S} C^{T} \mathcal{T}^{T} \Delta(t) C x(t) \\
& +2 e^{T}(t) \mathcal{S} C^{T} \mathcal{T}^{T} \mathcal{L}\left(e_{y}, t\right) e_{y}(t)
\end{aligned}
$$

Additionally, applying the inequality (3.12) to the second term on the right hand side of (3.31) we obtain

$$
\begin{aligned}
\frac{d}{d t} \mathcal{V}(e, t) \leq & e^{T}(t)\left(\mathcal{S} A_{K}+A_{K}^{T} \mathcal{S}\right) e(t)+\frac{1}{\gamma} e^{T}(t) \mathcal{S} C^{T} \mathcal{T}^{T} \mathcal{T} C \mathcal{S} e(t)+\gamma y^{T}(t) y(t) \\
& +2 e^{T}(t) \mathcal{S} C^{T} \mathcal{T}^{T} \mathcal{L}\left(e_{y}, t\right) e_{y}(t)
\end{aligned}
$$

Now by using the LMIs (3.29), the variable gain matrix (3.30) and the inequality (3.15), we have

$$
\begin{aligned}
\frac{d}{d t} \mathcal{V}(e, t) & \leq-e^{T}(t) \mathcal{Q} e(t)+\sigma(t) \\
& \leq-\zeta\|e(t)\|^{2}+\sigma(t)
\end{aligned}
$$

where $\zeta$ is a positive scalar given by $\zeta=\lambda_{\max }\{\mathcal{Q}\}$.

Therefore, one can see from the definition of the quadratic function $\mathcal{V}(e, t)$ and Proof 1 that the rest of proof of Theorem 2 is straightforward.

\subsection{Illustrative examples}

Consider the uncertain linear system described by

$$
\begin{aligned}
\frac{d}{d t} x(t) & =\left(\begin{array}{rrr}
-2.0 & 0.0 & -6.0 \\
0.0 & 1.0 & 1.0 \\
3.0 & 0.0 & -7.0
\end{array}\right) x(t)+\left(\begin{array}{l}
2.0 \\
1.0 \\
0.0
\end{array}\right) \Delta(t)\left(\begin{array}{lll}
1.0 & 0.0 & 1.0 \\
0.0 & 3.0 & 1.0
\end{array}\right) x(t)+\left(\begin{array}{l}
2.0 \\
1.0 \\
0.0
\end{array}\right) u(t) \\
y(t) & =\left(\begin{array}{lll}
1.0 & 0.0 & 0.0 \\
0.0 & 1.0 & 0.0
\end{array}\right) x(t)
\end{aligned}
$$

Namely, the matrix $\mathcal{T} \in \mathbb{R}^{1 \times 2}$ in the assumption (3.2) can be expressed as $\mathcal{T}=\left(\begin{array}{ll}2.0 & 1.0\end{array}\right)$.

Firstly, we design an output feedback gain matrix $K \in \mathbb{R}^{1 \times 2}$ for the nominal system. By selecting the design parameter $\alpha$ such as $\alpha=4.5$ and applying the LMI-based design algorithm (see. (2) and Appendix in (25)), we obtain the following output feedback gain matrix $K \in \mathbb{R}^{1 \times 2}$.

$$
K=\left(3.17745 \times 10^{-1}-1.20809 \times 10^{1}\right)
$$

Finally, we use Theorem 1 to design the proposed variable gain robust output feedback controller, i.e. we solve the LMIs (3.7). By selecting the symmetric positive definite matrix $\mathcal{Q} \in \mathbb{R}^{3 \times 3}$ such as $\mathcal{Q}=0.1 \times I_{3}$, we have

$$
\begin{aligned}
& \mathcal{S}=\left(\begin{array}{ccc}
7.18316 & 1.10208 & 3.02244 \times 10^{-1} \\
\star & 5.54796 & -6.10321 \times 10^{-2} \\
\star & \star & 4.74128
\end{array}\right) \\
& \gamma_{1}=2.01669 \times 10^{3}, \gamma_{2}=6.34316 \times 10^{2} \text {, } \\
& \Theta=\left(\begin{array}{cc}
3.14338 \times 10^{1} & 1.54786 \times 10^{1} \\
\star & 8.20347
\end{array}\right), \Psi=\left(\begin{array}{cc}
6.73050 & 6.45459 \\
\star & 6.57618
\end{array}\right)
\end{aligned}
$$




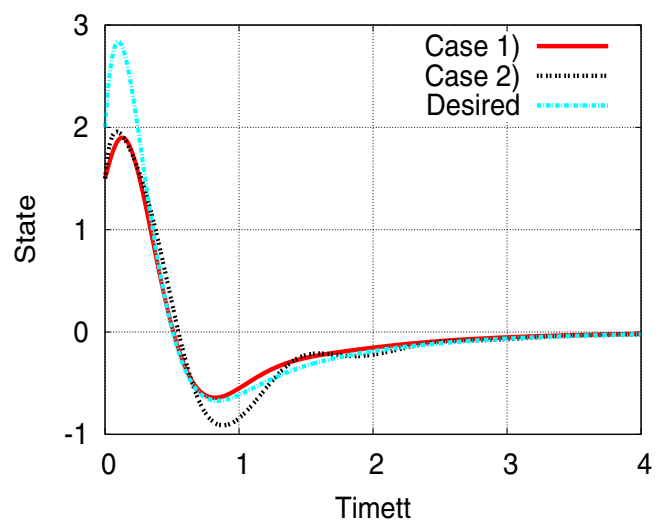

Fig. 4. Time histories of the state $x_{1}(t)$

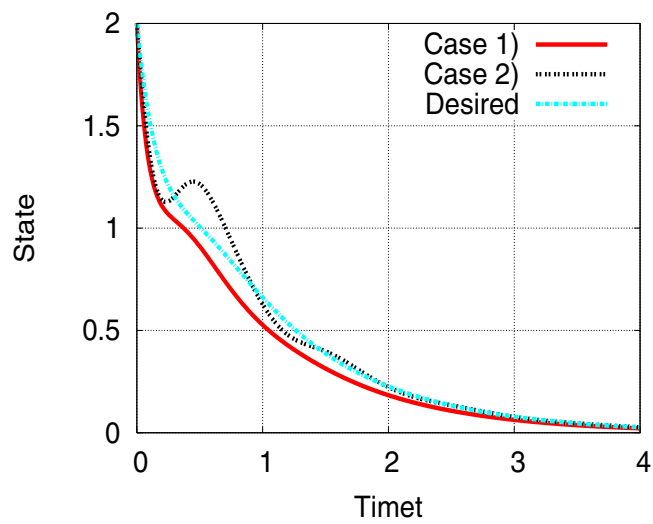

Fig. 5. Time histories of the state $x_{2}(t)$

In this example, we consider the following two cases for the unknown parameter $\Delta(t) \in \mathbb{R}^{1 \times 2}$.

- Case 1): $\Delta(t)=(7.30192-5.00436) \times 10^{-1}$

- Case 2$): \Delta(t)=(\sin (5 \pi t) \cos (5 \pi t))$

Furthermore, initial values for the uncertain system (3.24) and the nominal system are selected as $x(0)=(1.52 .0-4.5)^{T}$ and $\bar{x}(0)=(2.02 .0-5.0)^{T}$, respectively. Besides, we choose $\sigma(t) \in$ $\mathbb{R}^{+}$in $(3.8)$ as $\sigma(t)=5.0 \times 10^{12} \times \exp \left(-1.0 \times 10^{-4} t\right)$.

The results of the simulation of this example are depicted in Figures 4-7. In these figures, "Case 1)" and "Case 2)" represent the time-histories of the state variables $x_{1}(t)$ and $x_{2}(t)$ and the control input $u(t)$ generated by the proposed variable gain robust output feedback controller, and "Desired" shows the desired time-response and the desired control input generated by the nominal system. From Figures 4-6, we find that the proposed variable gain robust output feedback controller stabilize the uncertain linear system (3.34) in spite of plant uncertainties and achieves good transient performance. 


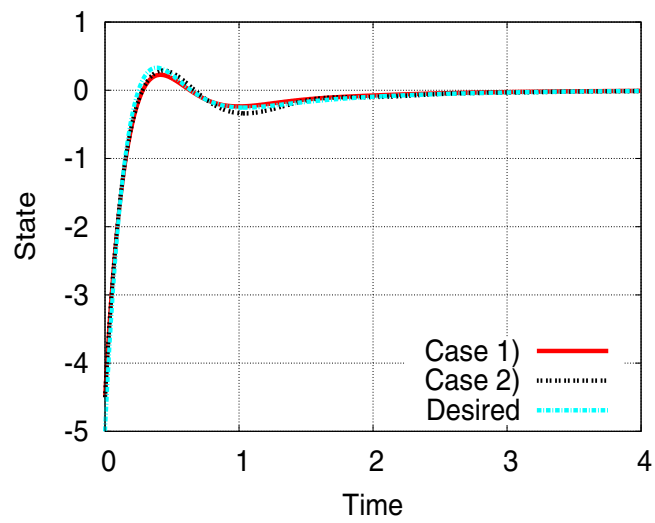

Fig. 6. Time histories of the state $x_{3}(t)$

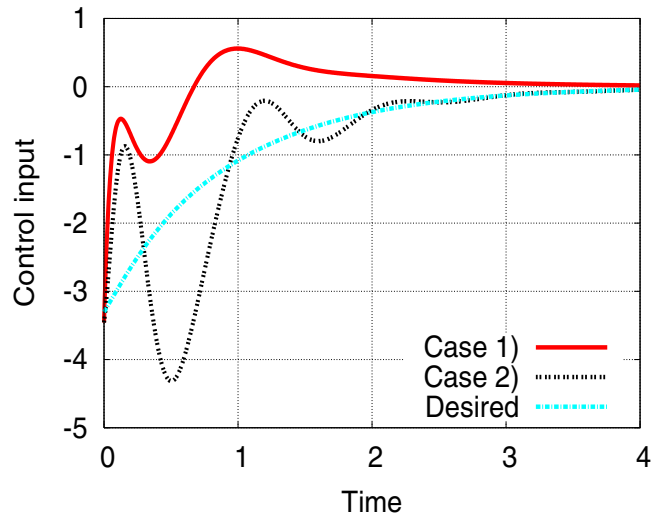

Fig. 7. Time histories of the control input $u(t)$

\subsection{Summary}

In this section, we have proposed a variable gain robust output feedback controller for a class of uncertain linear systems. Besides, by numerical simulations, the effectiveness of the proposed controller has been presented.

The proposed controller design method is easy to design a robust output feedback controller. Additionally, the proposed control scheme is adaptable when some assumptions are satisfied, and in cases where only the output signal of the controlled system is available, the proposed method can be used widely. In addition, the proposed controller is more effective for systems with larger uncertainties. Namely, for the upper bound on the perturbation region of the unknown parameter $\Delta(t)$ is larger than 1 , the proposed variable gain output feedback controller can easily be extended.

\section{Variable gain robust controllers based on piecewise Lyapunov functions}

The quadratic stability approach is popularly used for robust stability analysis of uncertain linear systems. This approach, however, may lead to conservative results. Alternatively, 
non-quadratic Lyapunov functions have been used to improve the estimate of robust stability and to design robust stabilizing controllers $(7 ; 30 ; 34)$. We have also proposed variable gain controllers and adaptive gain controllers based on Piecewise Lyapunov functions (PLFs) for a class of uncertain linear systems $(23 ; 24)$. However, the resulting variable gain robust controllers may occur the chattering phenomenon. In this section, we propose a variable gain robust state feedback controller avoiding chattering phenomenon for a class of uncertain linear systems via PLFs and show that sufficient conditions for the existence of the proposed variable gain robust state feedback controller.

\subsection{Problem formulation}

Consider a class of linear systems with non-linear perturbations represented by the following state equation (see Remark 2).

$$
\frac{d}{d t} x(t)=(A+\mathcal{D} \Delta(t) \mathcal{E}) x(t)+B u(t)
$$

where $x(t) \in \mathbb{R}^{n}$ and $u(t) \in \mathbb{R}^{m}$ are the vectors of the state (assumed to be available for feedback) and the control input, respectively. In (4.1), the matrices $A$ and $B$ denote the nominal values of the system, and the matrix $B$ has full column rank. The matrices $\mathcal{D}$ and $\mathcal{E}$ which have appropriate dimensions represent the structure of uncertainties. The matrix $\Delta(t) \in \mathbb{R}^{p \times q}$ represents unknown time-varying parameters and satisfies the relation $\|\Delta(t)\| \leq 1$. Note that the uncertain term $\mathcal{D} \Delta(t) \mathcal{E}$ consists of matched part and unmatched one. Additionally, introducing the integer $\mathcal{N} \in \mathbb{Z}^{+}$defined as

$$
\mathcal{N} \triangleq \arg \min _{\mathcal{Z} \in \mathbb{Z}^{+}}\{\mathcal{Z} \mid(\mathcal{Z} m-n) \geq 0\}
$$

we assume that there exist symmetric positive definite matrices $\mathcal{S}_{k} \in \mathbb{R}^{n \times n}(k=1, \cdots, \mathcal{N})$ which satisfies the following relation $(23 ; 24)$.

$$
\bigcap_{k=1}^{\mathcal{N}} \Omega_{\mathcal{S}_{k}}=\{0\}
$$

where $\Omega_{\mathcal{S}_{k}}$ represents a subspace defined as

$$
\Omega_{\mathcal{S}_{k}} \triangleq\left\{x \in \mathbb{R}^{n} \mid B^{T} \mathcal{S}_{k} x=0\right\}
$$

The nominal system, ignoring the unknown parameter in (4.1), is given by

$$
\frac{d}{d t} \bar{x}(t)=A \bar{x}(t)+B \bar{u}(t)
$$

where $\bar{x}(t) \in \mathbb{R}^{n}$ and $\bar{u}(t) \in \mathbb{R}^{m}$ are the vectors of the state and the control input, respectively. First of all, we adopt the standard linear quadratic LQ control theory for the nominal system (4.5) in order to generate the desirable transient response for the plant systematically, i.e. the control input is given by $\bar{u}(t)=K \bar{x}(t)$. Note that some other design method so as to generate the desired response for the controlled system can also be used (e.g. pole assignment). Thus the feedback gain matrix $K \in \mathbb{R}^{m \times n}$ is derived as $K=-\mathcal{R}^{-1} B^{T} \mathcal{P}$ where $\mathcal{P} \in \mathbb{R}^{n \times n}$ is unique solution of the algebraic Riccati equation

$$
A^{T} \mathcal{P}+\mathcal{P} A-\mathcal{P} B \mathcal{R}^{-1} B^{T} \mathcal{P}+\mathcal{Q}=0
$$


In (4.6), the matrices $\mathcal{Q} \in \mathbb{R}^{n \times n}$ and $\mathcal{R} \in \mathbb{R}^{m \times m}$ are design parameters and $\mathcal{Q}$ is selected such that the pair $(A, \mathcal{C})$ is detectable, where $\mathcal{C}$ is any matrix satisfying $\mathcal{Q}=\mathcal{C C}^{T}$, and then the matrix $A_{K} \triangleq A+B K$ is stable.

Now on the basis of the works of Oya et al.(21; 22), in order to obtain on-line information on the parameter uncertainty, we introduce the error vector $e(t) \triangleq x(t)-\bar{x}(t)$. Beside, using the optimal gain matrix $K \in \mathbb{R}^{m \times n}$ for the nominal system (4.5), we consider the following control input.

$$
u(t) \triangleq K x(t)+\psi(x, e, \mathcal{L}, t)
$$

where $\psi(x, e, \mathcal{L}, t) \in \mathbb{R}^{m}$ is a compensation input so as to reduce the effect of uncertainties and nonlinear perturbations, and it is supposed to have the following structure.

$$
\psi(x, e, \mathcal{L}, t) \triangleq \mathcal{F} e(t)+\mathcal{L}(x, e, t) e(t)
$$

where $\mathcal{F} \in \mathbb{R}^{m \times n}$ is a fixed gain matrix and $\mathcal{L}(x, e, t) \in \mathbb{R}^{m \times n}$ is an adjustable time-varying matrix. From (4.1), (4.5), (4.7) and (4.8), we have

$$
\begin{aligned}
\frac{d}{d t} e(t) & =(A+\mathcal{D} \Delta(t) \mathcal{E}) x(t)+B\{K x(t)+\psi(x, e, \mathcal{L}, t)\} \\
& =A_{\mathcal{F}} e(t)+\mathcal{D} \Delta(t) \mathcal{E} x(t)+B \mathcal{L}(x, e, t) e(t)
\end{aligned}
$$

In (4.9), $A_{\mathcal{F}} \in \mathbb{R}^{n \times n}$ is a matrix given by $A_{\mathcal{F}} \triangleq A_{K}+B \mathcal{F}$. Note that if asymptotical stability of the uncertain error system (4.9) is ensured, then the uncertain system (4.1) is robustly stable, because $e(t) \triangleq x(t)-\bar{x}(t)$. Here, the fixed gain matrix $\mathcal{F} \in \mathbb{R}^{m \times n}$ is determined by using LQ control theory for the nominal error system. Namely $\mathcal{F}=-\mathcal{R}_{\mathcal{F}} B^{T} \mathcal{X}_{\mathcal{F}}$ and $\mathcal{X}_{\mathcal{F}} \in \mathbb{R}^{n \times n}$ is unique solution of the algebraic Riccati equation

$$
A_{K}^{T} \mathcal{X}_{\mathcal{F}}+\mathcal{X}_{\mathcal{F}} A_{K}-\mathcal{X}_{\mathcal{F}} B \mathcal{R}_{\mathcal{F}}^{-1} B^{T} \mathcal{X}_{\mathcal{F}}+\mathcal{Q}_{\mathcal{F}}=0
$$

where $\mathcal{Q}_{\mathcal{F}} \in \mathbb{R}^{n \times n}$ and $\mathcal{R}_{\mathcal{F}} \in \mathbb{R}^{m \times m}$ are design parameters and symmetric positive definite matrices. A decision method of the time-varying matrix $\mathcal{L}(x, e, t) \in \mathbb{R}^{m \times n}$ will be stated in the next subsection.

From the above discussion, our control objective in this section is to design the robust stabilizing controller for the uncertain error system (4.9). That is to design the variable gain matrix $\mathcal{L}(x, e, t) \in \mathbb{R}^{m \times n}$ that the error system with uncertainties (4.9) is asymptotically stable.

\subsection{Synthesis of variable gain robust state feedback controllers via PLFs}

The following theorem gives sufficient conditions for the existence of the proposed controller.

Theorem 4. Consider the uncertain error system (4.9) and the control input (4.7) and (4.8).

Suppose that the matrices $\mathcal{S}_{k} \triangleq \mathcal{P}_{1}+\mathcal{P}_{2}+\cdots+\mathcal{P}_{\mathcal{N}}+\mathcal{P}_{k} B B^{T} \mathcal{P}_{k}(k=1, \cdots, \mathcal{N})$ satisfy the relation (4.3), where $\mathcal{P}_{k} \in \mathbb{R}^{n \times n}$ are symmetric positive definite matrices ${ }^{\dagger}$ satisfying the matrix inequalities

$$
\begin{aligned}
& \left(\mathcal{P}_{1}+\mathcal{P}_{2}+\cdots+\mathcal{P}_{\mathcal{N}}+\mathcal{P}_{k} B B^{T} \mathcal{P}_{k}\right) A_{\mathcal{F}}+A_{\mathcal{F}}^{T}\left(\mathcal{P}_{1}+\mathcal{P}_{2}+\cdots+\mathcal{P}_{\mathcal{N}}+\mathcal{P}_{k} B B^{T} \mathcal{P}_{k}\right) \\
& +\sum_{j=1}^{\mathcal{N}-1} \gamma_{j}{ }^{(k)} \mathcal{P}_{k} B B^{T} \mathcal{P}_{k}+\mathcal{Q}_{k}<0 \quad(k=1, \cdots, \mathcal{N})
\end{aligned}
$$

\footnotetext{
$\overline{{ }^{\dagger} \text { i.e. } \mathcal{S}_{k} \in \mathbb{R}^{n} \times n}$ are symmetric positive definite matrices.
} 
In (4.11), $\gamma_{j}^{(k)}(k=1, \cdots, \mathcal{N}, j=1, \cdots, \mathcal{N}-1)$ are positive scalars and $\mathcal{Q}_{k} \in \mathbb{R}^{n \times n}$ are symmetric positive definite matrices.

By using the matrices $\mathcal{S}_{k} \in \mathbb{R}^{n \times n}, \mathcal{L}(x, e, t) \in \mathbb{R}^{m \times n}$ is determined as

$$
\begin{gathered}
\mathcal{L}(x, e, t)=-\frac{\left(\left\|\mathcal{D}^{T} \mathcal{S}_{k} e(t)\right\|\|\mathcal{E} x(t)\|\right)^{2}}{\left(\sigma(t)+\left\|\mathcal{D}^{T} \mathcal{S}_{k} e(t)\right\|\|\mathcal{E} x(t)\|\right)\left\|B^{T} \mathcal{S}_{k} e(t)\right\|^{2}} B^{T} \mathcal{S}_{k} \\
\text { for } k=\arg \max _{k}\left\{e^{T}(t) \mathcal{P}_{k} B B^{T} \mathcal{P}_{k} e(t)\right\}
\end{gathered}
$$

In (4.12), $\sigma(t) \in \mathbb{R}^{1}$ is any positive uniform continuous and bounded function which satisfies

$$
\int_{t_{0}}^{t} \sigma(\tau) d \tau \leq \sigma^{*}<\infty
$$

where $\sigma^{*}$ is any positive constant and $t_{0}$ denotes an initial time. Then the uncertain error system (4.9) are bounded and

$$
\lim _{t \rightarrow \infty} e\left(t ; t_{0}, e\left(t_{0}\right)\right)=0
$$

Namely, asymptotical stability of the uncertain error system (4.9) is ensured.

Proof. Using symmetric positive definite matrices $\mathcal{P}_{k} \in \mathbb{R}^{n \times n} \quad(k=1, \cdots, \mathcal{N})$ which satisfy (4.11), we introduce the following piecewise quadratic function.

$$
\begin{aligned}
\mathcal{V}(e, t) & =e^{T}(t) \mathcal{S}_{k} e(t) \quad \text { for } \quad k=\arg \max _{k}\left\{e^{T}(t) \mathcal{P}_{k} B B^{T} \mathcal{P}_{k} e(t)\right\} \text { and } k=1, \cdots, \mathcal{N} \\
& =\max _{k}\left\{e^{T}(t) \mathcal{S}_{k} e(t)\right\}
\end{aligned}
$$

Note that the piecewise quadratic function $\mathcal{V}(e, t)$ is continuous and its level set is closed. The time derivative of the piecewise quadratic function $\mathcal{V}(e, t)$ can be written as

$$
\begin{gathered}
\frac{d}{d t} \mathcal{V}(e, t)=e^{T}(t)\left(\mathcal{S}_{k} A_{\mathcal{F}}+A_{\mathcal{F}}^{T} \mathcal{S}_{k}\right) e(t)+2 e^{T}(t) \mathcal{S}_{k} \mathcal{D} \Delta(t) \mathcal{E} x(t)+2 e^{T}(t) \mathcal{S}_{k} B \mathcal{L}(x, e, t) e(t) \\
\text { for } k=\arg \max _{k}\left\{e^{T}(t) \mathcal{P}_{k} B B^{T} \mathcal{P}_{k} e(t)\right\}
\end{gathered}
$$

Now, using Lemma 1, we can obtain

$$
\begin{aligned}
\frac{d}{d t} \mathcal{V}(e, t) \leq & e^{T}(t)\left(\mathcal{S}_{k} A_{\mathcal{F}}+A_{\mathcal{F}}^{T} \mathcal{S}_{k}\right) e(t)+2\left\|\mathcal{D}^{T} \mathcal{S}_{k} e(t)\right\|\|\mathcal{E} x(t)\| \\
& +2 e^{T}(t) \mathcal{S}_{k} B \mathcal{L}(x, e, t) e(t) \quad \text { for } \quad k=\arg \max _{k}\left\{e^{T}(t) \mathcal{P}_{k} B B^{T} \mathcal{P}_{k} e(t)\right\}
\end{aligned}
$$

Also, using the time-varying gain matrix (4.12) and the relation (4.17) and some trivial manipulations give the following relation for the time derivative of the piecewise quadratic 
function $\mathcal{V}(e, t)$.

$$
\begin{aligned}
\frac{d}{d t} \mathcal{V}(e, t) \leq & e^{T}(t)\left(\mathcal{S}_{k} A_{\mathcal{F}}+A_{\mathcal{F}}^{T} \mathcal{S}\right) e(t)+2\left\|\mathcal{D}^{T} \mathcal{S}_{k} e(t)\right\|\|\mathcal{E} x(t)\| \\
& +2 e^{T}(t) \mathcal{S}_{k} B\left\{-\frac{\left(\left\|\mathcal{D}^{T} \mathcal{S}_{k} e(t)\right\|\|\mathcal{E} x(t)\|\right)^{2}}{\left(\sigma(t)+\left\|\mathcal{D}^{T} \mathcal{S}_{k} e(t)\right\|\|\mathcal{E} x(t)\|\right)\left\|B^{T} \mathcal{S}_{k} e(t)\right\|^{2}} B^{T} \mathcal{S}_{k}\right\} e(t) \\
& \text { for } k=\arg \max _{k}\left\{e^{T}(t) \mathcal{P}_{k} B B^{T} \mathcal{P}_{k} e(t)\right\} \\
\leq & e^{T}(t)\left(\mathcal{S}_{k} A_{\mathcal{F}}+A_{\mathcal{F}}^{T} \mathcal{S}\right) e(t)+\sigma(t) \quad \text { for } \quad k=\arg \max _{k}\left\{e^{T}(t) \mathcal{P}_{k} B B^{T} \mathcal{P}_{k} e(t)\right\}
\end{aligned}
$$

Now we consider the following inequality.

$$
e^{T}(t)\left(\mathcal{S}_{k} A_{\mathcal{F}}+A_{\mathcal{F}}^{T} \mathcal{S}_{k}\right) e(t)<0 \quad \text { for } \quad k=\arg \max _{k}\left\{e^{T}(t) \mathcal{P}_{k} B B^{T} \mathcal{P}_{k} e(t)\right\}
$$

By using Lemma 4 (S-procedure), the inequality (4.19) is satisfied if and only if there exist $\mathcal{S}_{k}>0$ and $\gamma_{j}^{(k)} \geq 0(j=1, \cdots, \mathcal{N}-1, k=1, \cdots, \mathcal{N})$ satisfying

$$
\begin{gathered}
\mathcal{S}_{1} A_{\mathcal{F}}+A_{\mathcal{F}}^{T} \mathcal{S}_{1}+\sum_{j=1}^{\mathcal{N}-1} \gamma_{j}^{(1)} \mathcal{P}_{1} B B^{T} \mathcal{P}_{1}-\gamma_{1}^{(1)} \mathcal{P}_{2} B B^{T} \mathcal{P}_{2}-\cdots-\gamma_{\mathcal{N}-1}^{(1)} \mathcal{P}_{\mathcal{N}} B B^{T} \mathcal{P}_{\mathcal{N}}<0 \\
\vdots \\
\mathcal{S}_{\mathcal{N}} A_{\mathcal{F}}+A_{\mathcal{F}}^{T} \mathcal{S}_{\mathcal{N}}+\sum_{j=1}^{\mathcal{N}-1} \gamma_{j}^{(\mathcal{N})} \mathcal{P}_{\mathcal{N}} B B^{T} \mathcal{P}_{\mathcal{N}}-\gamma_{1}^{(\mathcal{N})} \mathcal{P}_{2} B B^{T} \mathcal{P}_{2}-\cdots-\gamma_{\mathcal{N}-1}^{(\mathcal{N})} \mathcal{P}_{\mathcal{N}-1} B B^{T} \mathcal{P}_{\mathcal{N}-1}<0
\end{gathered}
$$

Noting that since the condition (4.11) is a sufficient condition for the matrix inequalities (4.20), if the inequalities (4.11) are satisfied, then the condition (4.20) is also satisfied. Therefore, we have the following relation.

$$
e^{T}(t)\left(\mathcal{S}_{k} A_{\mathcal{F}}+A_{\mathcal{F}}^{T} \mathcal{S}_{k}\right) e(t)<-e^{T}(t) \mathcal{Q}_{k} e(t)
$$

Besides, by letting $\zeta_{k} \triangleq \min _{k}\left\{\lambda_{\min }\left\{\mathcal{Q}_{k}\right\}\right\}$, we obtain

$$
\frac{d}{d t} \mathcal{V}(e, t) \leq-\zeta_{k}\|e(t)\|^{2}+\sigma(t) \quad \text { for } \quad k=\arg \max _{k}\left\{e^{T}(t) \mathcal{P}_{k} B B^{T} \mathcal{P}_{k} e(t)\right\}
$$

On the other hand, from the definition of the piecewise quadratic function, there always exist two positive constants $\delta_{\min }$ and $\delta_{\max }$ such that for any $t \geq t_{0}$,

$$
\eta^{-}(\|e(t)\|) \leq \mathcal{V}(e, t) \leq \eta^{+}(\|e(t)\|)
$$

where $\eta^{-}(\|e(t)\|)$ and $\eta^{+}(\|e(t)\|)$ are given by

$$
\begin{aligned}
& \eta^{-}(\|e(t)\|) \triangleq \delta_{\min }\|e(t)\|^{2} \\
& \eta^{+}(\|e(t)\|) \triangleq \delta_{\max }\|e(t)\|^{2}
\end{aligned}
$$


It is obvious that any solution $e\left(t ; t_{0}, e\left(t_{0}\right)\right)$ of the error system is continuous. In addition, it follows from (4.22) and (4.23), that for any $t \geq t_{0}$, the following relation holds.

$$
\begin{aligned}
& 0 \leq \eta^{-}(\|e(t)\|) \leq \mathcal{V}(e, t)=\mathcal{V}\left(e, t_{0}\right)+\int_{t_{0}}^{t} \frac{d}{d t} \mathcal{V}(e, \tau) d \tau \\
& \mathcal{V}\left(e, t_{0}\right)+\int_{t_{0}}^{t} \frac{d}{d t} \mathcal{V}(e, \tau) d \tau \leq \eta^{+}\left(\left\|e\left(t_{0}\right)\right\|\right)-\int_{t_{0}}^{t} \eta^{*}(\|e(\tau)\|) d \tau+\int_{t_{0}}^{t} \sigma(\tau) d \tau
\end{aligned}
$$

In (4.25), $\eta^{*}(\|e(t)\|)$ is defined as

$$
\eta^{*}(\|e(t)\|) \triangleq \zeta_{k}\|e(t)\|^{2}
$$

Therefore, from (4.25) we can obtain the following two results. Firstly, taking the limit as $t$ approaches infinity on both sides of the inequality (4.25), we have

$$
0 \leq \eta^{+}\left(\left\|e\left(t_{0}\right)\right\|\right)-\lim _{t \rightarrow \infty} \int_{t_{0}}^{t} \eta^{*}(\|e(\tau)\|) d \tau+\lim _{t \rightarrow \infty} \int_{t_{0}}^{t} \sigma(\tau) d \tau
$$

Thus one can see from (4.13) and (4.27) that

$$
\lim _{t \rightarrow \infty} \int_{t_{0}}^{t} \eta^{*}(\|e(\tau)\|) d \tau \leq \eta^{+}\left(\left\|e\left(t_{0}\right)\right\|\right)+\sigma^{*}
$$

On the other hand, from (4.25), we obtain

$$
0 \leq \eta^{-}(\|e(t)\|) \leq \eta^{+}\left(\left\|e\left(t_{0}\right)\right\|\right)+\int_{t_{0}}^{t} \sigma(\tau) d \tau
$$

It follows from (4.13) and (4.29) that

$$
0 \leq \eta^{-}(\|e(t)\|) \leq \eta^{+}\left(\left\|e\left(t_{0}\right)\right\|\right)+\sigma^{*}
$$

The relation (4.30) implies that $e(t)$ is uniformly bounded. Since $e(t)$ has been shown to be continuous, it follows that $e(t)$ is uniformly continuous. Therefore, one can see from the definition that $\eta^{*}(\|e(t)\|)$ is also uniformly continuous. Applying the Lemma 3 ( Barbalat's lemma ) to (4.28) yields

$$
\lim _{t \rightarrow \infty} \eta^{*}(\|e(t)\|)=\lim _{t \rightarrow \infty} \zeta_{k}\|e(t)\|=0
$$

Namely, asymptotical stability of the uncertain error system (4.9) is ensured. Thus the uncertain linear system (4.1) is also stable.

Thus the proof of Theorem 4 is completed.

Remark 2. In this section, we consider the uncertain dynamical system (4.1) which has uncertainties in the state matrix only. The proposed robust controller can also be applied to the case that the uncertainties are included in both the system matrix and the input one. By introducing additional actuator dynamics and constituting an augmented system, uncertainties in the input matrix are embedded in the system matrix of the augmented system(36). Therefore the same design procedure can be applied. 
Remark 3. In order to get the proposed controller, symmetric positive definite matrices $\mathcal{S}_{k} \in$ $\mathbb{R}^{n \times n}(k=1, \cdots, \mathcal{N})$ satisfying the assumption (4.3) are required. The condition (4.3) is reduced to the following rank condition.

$$
\operatorname{rank}\left\{\left(\mathcal{S}_{1} B \mathcal{S}_{2} B \cdots \mathcal{S}_{\mathcal{N}} B\right)^{T}\right\}=n
$$

However there is not a globally effective method to obtain matrices satisfying the conditions (4.32). In future work, we will examine the assumption (4.3) and the condition (4.32).

Remark 4. In this section, we introduce the compensation input (4.8). From (4.8) and (4.12), one can see that if $e(t)=0$, then the relation $\psi(x, e, \mathcal{L}, t) \equiv 0$ is satisfied. Beside, we find that the variable gain matrix $\mathcal{L}(x, e, t) \in \mathbb{R}^{m \times n}$ can be calculated except for $e(t)=0$ (see (24)).

Now, we consider the condition (4.11) in Theorem 4 . The condition (4.11) requires symmetric positive definite matrices $\mathcal{P}_{k} \in \mathbb{R}^{n \times n}$ and positive scalars $\gamma_{j}^{(k)} \in \mathbb{R}^{1}$ for stability. In this section, on the basis of the works of Oya et al.(23; 24), we consider the following inequalities instead of (4.11).

$$
\begin{aligned}
& \left(\mathcal{P}_{1}+\mathcal{P}_{2}+\cdots+\mathcal{P}_{\mathcal{N}}\right) A_{\mathcal{F}}+A_{\mathcal{F}}^{T}\left(\mathcal{P}_{1}+\mathcal{P}_{2}+\cdots+\mathcal{P}_{\mathcal{N}}\right) \\
& +\sum_{j=1}^{\mathcal{N}-1} \gamma_{j}^{(k)} \mathcal{P}_{k} B B^{T} \mathcal{P}_{k}+\mathcal{Q}_{k}<0 \quad(k=1, \cdots, \mathcal{N})
\end{aligned}
$$

In addition, introducing complementary variables $\xi_{j}^{(k)} \triangleq\left(\gamma_{j}^{(k)}\right)^{-1}(j=1, \cdots, \mathcal{N}-1, k=$ $1, \cdots, \mathcal{N})$ and using Lemma 3 (Schur complement), we find that the condition (4.33) equivalent to the following LMIs.

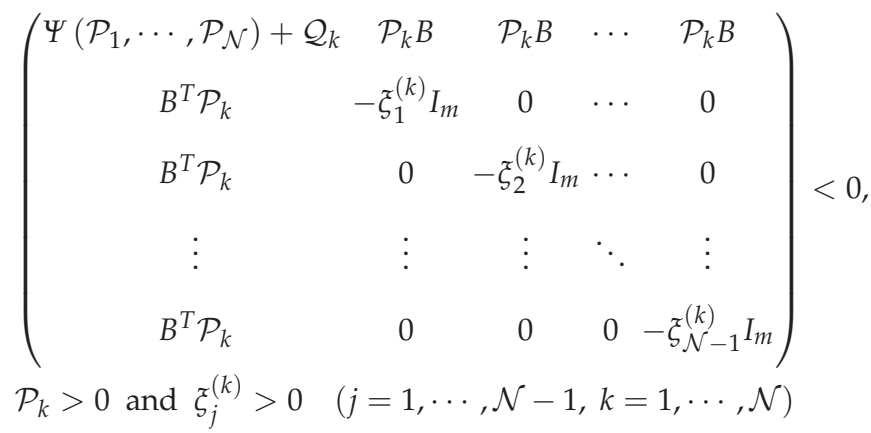

where $\Psi\left(\mathcal{P}_{1}, \cdots, \mathcal{P}_{\mathcal{N}}\right)$ in $(1,1)$-block of the LMIs $(4.34)$ is given by

$$
\Psi\left(\mathcal{P}_{1}, \cdots, \mathcal{P}_{\mathcal{N}}\right)=\left(\mathcal{P}_{1}+\mathcal{P}_{2}+\cdots+\mathcal{P}_{\mathcal{N}}\right) A_{\mathcal{F}}+A_{\mathcal{F}}^{T}\left(\mathcal{P}_{1}+\mathcal{P}_{2}+\cdots+\mathcal{P}_{\mathcal{N}}\right)
$$

Note that if there exist symmetric positive definite matrices $\mathcal{P}_{k} \in \mathbb{R}^{n \times n}$ and positive scalars $\gamma_{j}^{(k)} \in \mathbb{R}^{1}$ which satisfy the matrix inequalities (4.34), then the matrix inequality condition (4.11) is also satisfied $(23 ; 24)$.

From the above discussion, one can see that in order to get the proposed robust controller, the positive scalars $\gamma_{j}^{(k)} \in \mathbb{R}^{1}$ and the symmetric positive definite matrices $\mathcal{P}_{k} \in \mathbb{R}^{n \times n}$ which satisfy the LMIs (4.34) and the assumption (4.3) are needed. Therefore firstly, we solve the LMIs (4.34) and next, we check the rank condition (4.32). 


\subsection{Illustrative examples}

Consider the following uncertain linear system, i.e. $\mathcal{Z}=2$.

$$
\frac{d}{d t} x(t)=\left(\begin{array}{rr}
-4 & 1 \\
0 & 2
\end{array}\right) x(t)+\left(\begin{array}{rr}
5 & -1 \\
0 & 1
\end{array}\right) \Delta(t)\left(\begin{array}{ll}
1 & 1 \\
0 & 1
\end{array}\right) x(t)+\left(\begin{array}{l}
0 \\
1
\end{array}\right) u(t)
$$

By applying Theorem 4 , we consider deriving the proposed robust controller. Now we select the weighting matrices $\mathcal{Q} \in \mathbb{R}^{2 \times 2}$ and $\mathcal{R} \in \mathbb{R}^{1 \times 1}$ such as $\mathcal{Q}=1.0 I_{2}$ and $\mathcal{R}=4.0$ for the quadratic cost function for the standard linear quadratic control problem for the nominal system, respectively. Then solving the algebraic Riccati equation (4.6), we obtain the optimal gain matrix

$$
K=\left(-5.15278 \times 10^{-3}-4.06405\right)
$$

In addition, setting the design parameters $\mathcal{Q}_{\mathcal{F}}$ and $\mathcal{R}_{\mathcal{F}}$ such as $\mathcal{Q}_{\mathcal{F}}=10.0 \times 10^{6} I_{2}$ and $\mathcal{R}_{\mathcal{F}}=$ 1.0 , respectively, we have the following fixed gain matrix.

$$
\mathcal{F}=(-1.23056-9.99806) \times 10^{3}
$$

Besides, selecting the matrices $\mathcal{Q}_{k}(k=1,2)$ in $(4.34)$ as

$$
\mathcal{Q}_{1}=\left(\begin{array}{cc}
20.0 & 1.0 \\
1.0 & 1.0
\end{array}\right), \quad \mathcal{Q}_{2}=\left(\begin{array}{cc}
1.0 & 0.0 \\
0.0 & 20.0
\end{array}\right)
$$

and solving the LMI condition (4.34), we get

$$
\begin{aligned}
& \mathcal{P}_{1}=\left(\begin{array}{cc}
7.59401 \times 10^{1} & 6.82676 \times 10^{-4} \\
6.82676 \times 10^{-4} & 2.00057 \times 10^{-3}
\end{array}\right) \\
& \mathcal{P}_{2}=\left(\begin{array}{cc}
7.59401 \times 10^{1} & 5.96286 \times 10^{-4} \\
5.96286 \times 10^{-4} & 5.76862 \times 10^{-2}
\end{array}\right) \\
& \gamma_{1}=7.13182 \times 10^{-3}, \gamma_{2}=7.13182 \times 10^{-3}
\end{aligned}
$$

From (4.36) and (4.40), $\Omega_{\mathcal{S}_{k}}(k=1,2)$ can be written as

$$
\begin{aligned}
& \Omega_{\mathcal{S}_{1}}=\left\{x \in \mathbb{R}^{2} \mid 1.28240 x_{1}+7.80246 x_{2}=0\right\} \\
& \Omega_{\mathcal{S}_{2}}=\left\{x \in \mathbb{R}^{2} \mid 1.28032 x_{1}+7.77319 x_{2}=0\right\}
\end{aligned}
$$

and thus the assumption (4.3) is satisfied.

On the other hand for the uncertain linear system (4.36), the quadratic stabilizing controller based on a fixed quadratic Lyapunov function cannot be obtained, because the solution of the LMI of (A.1) does not exist.

In this example, we consider the following two cases for the unknown parameter $\Delta(t)$.

- Case 1) $: \Delta(t)=\left(\begin{array}{r}-4.073608 .06857 \\ 4.413793 .81654\end{array}\right) \times 10^{-1}$ 
- Case 2) $: \Delta(t)=\left(\begin{array}{cc}\cos (3.0 \pi t) & 0 \\ 0 & -\sin (3.0 \pi t)\end{array}\right)$

Besides, for numerical simulations, the initial values for the uncertain linear system (4.36)

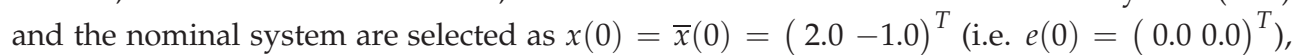
respectively, and we choose $\sigma(t) \in \mathbb{R}^{+}$in (4.12) as $\sigma(t)=5.0 \times 10^{12} \times \exp \left(-1.0 \times 10^{-3} t\right)$. The results of the simulation of this example are depicted in Figures 8-10. In these Figures, "Case 1)" and "Case 2)" represent the time-histories of the state variables $x_{1}(t)$ and $x_{2}(t)$ and the control input $u(t)$ for the proposed variable gain robust controller. "Desired" shows the desired time-response and the desired control input generated by the nominal system.

From Figures 8-10, we find that the proposed robust controller stabilizes the uncertain system (4.36) in spite of uncertainties. one can see from Figure 10 the proposed controller can avoid serious chattering. Therefore the effectiveness of the proposed controller is shown.

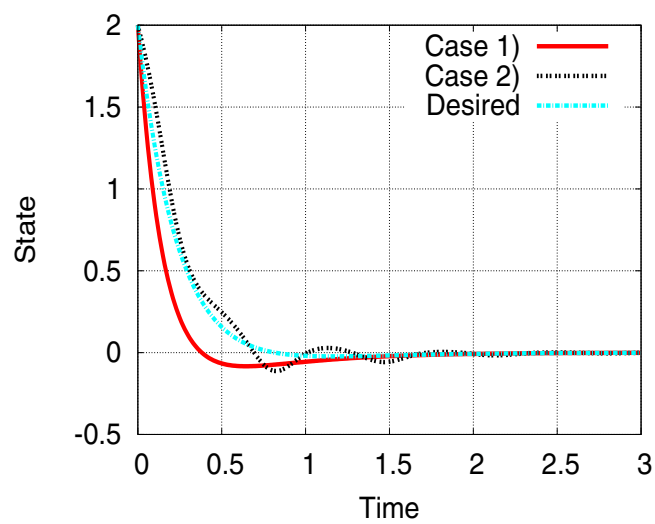

Fig. 8. Time histories of the state $x_{1}(t)$

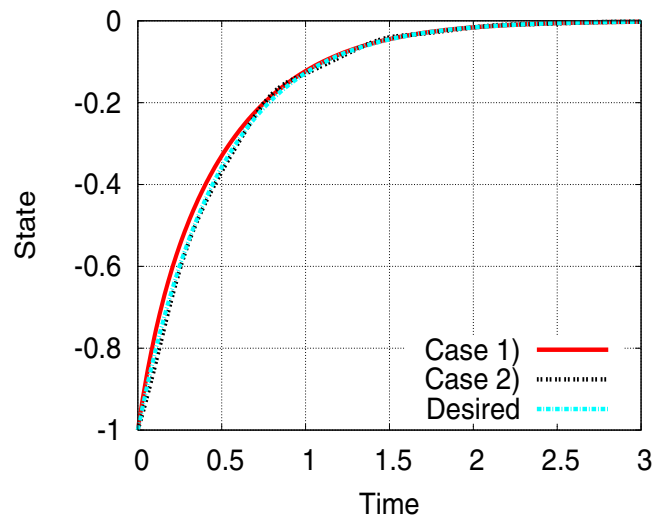

Fig. 9. Time histories of the state $x_{2}(t)$ 


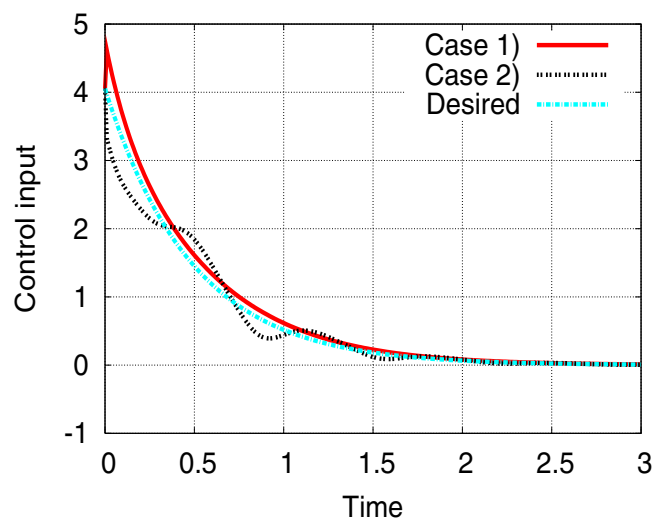

Fig. 10. Time histories of the control input $u(t)$

\subsection{Summary}

In this section, we have proposed a design method of a variable gain robust controller for a class of uncertain nonlinear systems. The uncertainties under consideration are composed of matched part and unmatched one, and by using the concept of piecewise Lyapunov functions, we have shown that the proposed robust controller can be obtained by solving LMIs (4.34) and cheking the rank condition (4.32). By numerical simulations, the effectiveness of the proposed controller has been presented.

\section{Conclusions and future works}

In this chapter, we have presented that the variable gain robust controller for a class of uncertain linear systems and through the numerical illustrations, the effectiveness of the proposed vaiable gain robust controllers has been shown. The advantage of the proposed controller synthesis is as follows; the proposed variable gain robust controller in which the real effect of the uncertainties can be reflected as on-line information is more flexible and adaptive than the conventional robust controller with a fixed gain which is derived by the worst-case design for the parameter variations. Additionally the proposed control systems are constructed by renewing the parameter which represents the perturbation region of unknown parameters, and there is no need to solve any other equation for the stability.

In Section 2 for linear systems with matched uncertainties, a design problem of variable gain robust state feedback controllers in order to achieve satisfactory transient behavior as closely as possible to desirable one generated by the nominal system is considered. Section 3 extends the result for the variable gain robust state feedback controller given in Section 2 to variable gain robust output feedback controllers. In this Section, some assumptions for the structure of the system parameters are introduced and by using these assumptions, an LMI-based the variable gain robust output feedback controller synthesis has been presented. In Section 4, the design method of variable gain robust state feedback controller via piecewise Lyapunov functions has been suggested. One can see that the crucial difference between the existing results and the proposed variable gain controller based on PLFs is that for uncertain linear systems which cannot be statilizable via the conventional quadratic stabilizing controllers, the proposed design procedure can stabilize it. Besides, it is obvious that the proposed variable robust control scheme is more effective for linear systems with larger uncertainties. 
The future research subjects are an extension of the variable gain robust state feedback controller via PLFs to output feedback control systems. Besides, the problem for the extension to such a broad class of systems as uncertain large-scale systems, uncertain time-delay systems and so on should be tackled. Furthermore in future work, we will examine the condition (3.2) in section 3 and assumptions (4.3) and (4.32) in section 4 .

On the other hand, the design of feedback controllers is often complicated by presence of physical constraints : saturating actuators, temperatures, pressures within safety margins and so on. If the constraints are violated, serious consequences may ensue, for example, physical components may be damaged, or saturation may cause a loss of closed-loop stability. In particular, input saturation is a common feature of control systems and the stabilization problems of linear systems with control input saturation have been studied (e.g. $(17 ; 32)$ ). Furthermore, some researchers have investigated analysis of constrained linear systems and reference managing for linear systems subject to input and state constraints (e.g. $(10 ; 15))$. Therefore, the future research subjects are to address the constrained robust control problems reducing the effect of unknown parameters.

\section{Appendix}

\subsection{Quadratic stabilization}

The following lemma provides a LMI-based design method of a robust controller via Lyapunov stability criterion.

Lemma A.1. Consider the uncertain linear system (4.1) and the control law $u(t)=H x(t)$.

There exists the state feedback gain matrix $H \in \mathbb{R}^{m \times n}$ such that the control law $u(t)=H x(t)$ is a quadratic stabilizing control, if there exist $\mathcal{X}>0, \mathcal{Y}$ and $\delta>0$ satisfying the LMI

$$
\left(\begin{array}{cc}
A \mathcal{X}+\mathcal{X} A^{T}+B \mathcal{Y}+\mathcal{Y}^{T} B^{T}+\delta \mathcal{D D} \mathcal{D}^{T} & \mathcal{X} \mathcal{E}^{T} \\
\mathcal{E} \mathcal{X} & -\delta I_{q}
\end{array}\right)<0
$$

If the solution $\mathcal{X}, \mathcal{Y}$ and $\delta$ of the LMI (A.1) exists, then the gain matrix $H \in \mathbb{R}^{m \times n}$ is obtained as $H=\mathcal{Y} \mathcal{X}^{-1}$.

Proof. Introducing the quadratic function $\mathcal{V}(x, t) \triangleq e x^{T}(t) \mathcal{P} x(t)$ as a Lyapunov function candidate, we have

$$
\begin{aligned}
\frac{d}{d t} \mathcal{V}(x, t) & =x^{T}(t)\left\{\mathcal{P}(A+B H)+(A+B H)^{T} \mathcal{P}\right\} x(t)+2 x^{T}(t) \mathcal{P} \mathcal{D} \Delta(t) \mathcal{E} x(t) \\
& \leq x^{T}(t)\left\{\mathcal{P}(A+B H)+(A+B H)^{T} \mathcal{P}\right\} x(t)+\delta x^{T}(t) \mathcal{P} \mathcal{D} \mathcal{D}^{T} \mathcal{P} x(t)+\frac{1}{\delta} x^{T}(t) \mathcal{E}^{T} \mathcal{E} x(t)
\end{aligned}
$$

Here we have used the well-known relation (3.12). Thus the uncrtain linear system (4.1) is robustly stable provided that the following relation is satisfied.

$$
\mathcal{P}(A+B H)+(A+B H)^{T} \mathcal{P}+\delta \mathcal{P} \mathcal{D} \mathcal{D}^{T} \mathcal{P}+\frac{1}{\delta} \mathcal{E}^{T} \mathcal{E}<0
$$

We introduce the matrix $\mathcal{X} \triangleq \mathcal{P}^{-1}$ and consider the change of variable $\mathcal{Y} \triangleq H \mathcal{X}$. Then, by preand post-multiplying (A.3) by $\mathcal{X}=\mathcal{P}^{-1}$, we have

$$
A \mathcal{X}+\mathcal{X} A^{T}+B \mathcal{Y}+\mathcal{Y}^{T} B^{T}+\delta \mathcal{D} \mathcal{D}^{T}+\frac{1}{\delta} \mathcal{X} \mathcal{E}^{T} \mathcal{E} \mathcal{X}<0
$$


One can see from Lemma 2 (Schur complement) that the inequaity (A.4) is equivalent to the LMI (A.1).

\section{References}

[1] H. L. S. Almeida, A. Bhaya and D. M. Falcão, "A Team Algorithm for Robust Stability Analysis and Control Design of Uncertain Time-Varying Systems using Piecewise Quadratic Lyapunov Functions", Int. J. Robust and Nonlinear Contr., Vol.11, No.1, pp.357-371, 2001.

[2] R. E. Benton, JR and D. Smith, "A Non-itarative LMI-based Algorithm for Robust Static-output-feedback Stabilization", Int. J. Contr., Vol.72, No.14, pp.1322-1330, 1999.

[3] B. R. Bermish , M. Corless and G. Leitmann, "A New Class of Stabilizing Controllers for Uncertain Dynamical Systems", SIAM J. Contr. Optimiz. Vol.21, No.2, pp.246-255, 1983.

[4] S. Boyd, L. El Ghaoui, E. Feron and V. Balakrishnan, Linear Matrix Inequalities in System and Control Theory, SIAM Studies in Applied Mathmatics, 1994.

[5] Y. K. Choi, M. J. Chung and Z. Bien, "An Adaptive Control Scheme for Robot Manipulators", Int. J. Contr., Vol.44, No.4, pp.1185-1191, 1986.

[6] J. C. Doyle, K. Glover, P. P. Khargonekar and B. A. Francis, "State-Space Solutions to Standarad $\mathcal{H}^{2}$ and $\mathcal{H}^{\infty}$ Control Problems", IEEE Trans. Automat. Contr., Vol.34, No.8, pp.831-847, 1989.

[7] P. Gahinet, P. Apkarian and M. Chilali, "Affine Parameter Dependent Lyapunov Functions and Real Parameter Uncertainty", IEEE Trans. Automat. Contr., Vol.41, No.3, pp.436-442, 1996.

[8] F. R. Gantmacher, "The Theory of Matrices", Vol.1, Chelsea Publishing Company, New York, 1960.

[9] J. C. Geromel, C. C. De Souza and R. E. Skelton, “LMI Numerical Solution for Output Feedbadck Stabilization", Proc. of the 1994 American Contr. Conf., Baltimore, MD, USA, pp.40-44, 1994.

[10] E. G. Gilbert and I. Kolmanovsky, "Nonlinear Tracking Controlin the Presence of State and Control Constraints: A Generalized Reference Govonor", Automatica, Vol.38, No.12, pp.2071-2077, 2002.

[11] T. Iwasaki, R. E. Skelton and J. C. Geromel, "Linear Quadratic Suboptimal Control with Static Output Feedback", Syst. \& Contr. Lett., Vol.23, No.6, pp.421-430, 1994.

[12] F. Jabbari and W. E. Schmitendolf, "Effect of Using Observers on Stabilization of Uncertain Linear Systems", IEEE Trans. Automat. Contr., Vol.38, No.2, pp.266-271, 1993.

[13] H. K. Khalil, "Nonlinear Systems, Third Edition", Prentice Hall, 2002.

[14] P. P. Khargonekar and M. A. Rotea, "Mixed $\mathcal{H}_{2} / \mathcal{H}_{\infty}$ Control , A Convex Optimization Approach", IEEE Trans. Automat. Contr., Vol.36, No.7, pp.824-837, 1991.

[15] K. Kogiso and K. Hirata, "Reference Governor for Contrained Systems with Time-Varying References", J. Robotics and Autonomous Syst., Vol.57, Issue 3, pp.289-295, 2009.

[16] V. Kučera and C. E. De Souza, "A Necessary and Sufficient Conditions for Output Feedback Stabilizability", Automatica, Vol.31, No.9, pp.1357-1359, 1995.

[17] Z. Lin, M. Pachter and S. Band, "Toward Improvement of Tracking Performance Nonlinear Feedback for Linear Systems", Int. J. Contr., Vol.70, No.1, pp.1-11 (1998)

[18] M. Maki and K. Hagino, "Robust Control with Adaptation Mechanism for Improving Transient Behavior", Int. J. Contr., Vol.72, No.13, pp.1218-1226, 1999. 
[19] H. Oya and K. Hagino: "Robust Servo System with Adaptive Compensation Input for Linear Uncertain Systems", Proc. of the 4th Asian Contr. Conf., pp.972-977, SINGAPORE, 2002.

[20] H. Oya and K. Hagino : "Observer-based Robust Control Giving Consideration to Transient Behavior for Linear Systems with Structured Uncertainties", Int. J. Contr., Vol.75, No.15, pp.1231-1240, 2002.

[21] H. Oya and K. Hagino, "Robust Control with Adaptive Compensation Input for Linear Uncertain Systems", IEICE Trans. Fundamentals of Electronics, Communications and Computer Sciences, Vol.E86-A, No.6, pp.1517-1524, 2003.

[22] H. Oya and K. Hagino, "Adaptive Robust Control Scheme for Linear Systems with Structured Uncertainties", IEICE Trans. Fundamentals of Electronics, Communications and Computer Sciences, Vol.E87-A, No.8, pp.2168-2173, 2004.

[23] H. Oya, K. Hagino, S. Kayo and M. Matsuoka, "Adaptive Robust Stabilization for a Class of Uncertain Linear Systems via Variable Gain Controllers", Proc. of the 45th IEEE Conf. on Decision and Contr., pp.1183-1188, San Diego, USA, 2006.

[24] H. Oya, K. Hagino and S. Kayo, "Adaptive Robust Control Based on Piecewise Lyapunov Functions for a Class of Uncertain Linear Systems", Proc. of the European Contr. Conf. 2007 (ECC2007), pp.810-815, Kos, GREECE, 2007.

[25] H. Oya, K. Hagino and S. Kayo, "Synthesis of Adaptive Robust Output Feedback Controllers for a Class of Uncertain Linear Systems", Proc. of the 47th IEEE Conf.on Decision and Contr., pp.995-1000, Cancun, MEXICO, 2008.

[26] H. Oya and K. Hagino, "A New Adaptive Robust Controller Avoiding Chattering Phenomenon for a Class of Uncertain Linear Systems", Proc. of the 28th IASTED Int. Conf. on Modeling, Identification and Contr., pp.236-241, Innsbruck, 2009.

[27] I. R. Petersen, "A Riccati Equation Approach to the Design of Stabilizing Controllers and Observers for a Class of Uncertain Linear Systems", IEEE Trans. Automat. Contr., Vol.30, No.9, pp.904-907, 1985.

[28] I. R. Petersen and D. C. McFarlane, “Optimal Guaranteed Cost Control and Filtering for Uncertain Linear Systems", IEEE Trans. Automat. Contr., Vol.39, No.9, pp.1971-1977, 1994.

[29] I. R. Petersen and C. C. Hollot, "A Riccati Equation Approach to the Stabilization of Uncertain Linear Systems", Automatica, Vol.22, No.4, pp.397-411, 1986.

[30] E. S. Pyatnitskii, V. I. Skrodinskii, "Numerical Methods of Lyapunov Function Contruction and Their Application to the Absolute Stability Problem", Syst. \& Contr. Lett., Vol.2, No.1, 1986.

[31] S. O. Reza Moheimani and I. R. Petersen : "Optimal Guaranteed Cost Control of Uncertain Systems via Static and Dynamic Output Feedback", Automatica, Vol.32, No.4, pp.575-579, 1996.

[32] M. C. Turner, I. Postlethwaite and D. J. Walker, "Non-linear Tracking Control for Multivariable Constrained Input Linear Systems", Int. J. Contr., Vol.73, No.12, pp.1160-1172, 2000.

[33] S. Ushida, S. Yamamoto and H. Kimura, "Quadratic Stabilization by $\mathcal{H}^{\infty}$ state feedback controllers with Adjustable Parameters", Proc. of the 35th IEEE Conf. Decision and Contr., pp.1003-1008, Kobe, JAPAN, 1996.

[34] V. Veselý, "Design of Robust Output Affine Quadratic Controller", Proc. of 2002 IFAC World Congress, pp.1-6, Barcelona, SPAIN, 2002. 
[35] L. Xie, S. Shishkin and M. Fu, "Piecewise Lyapunov Functions for Robust Stability of Linear Time-Varying Systems", Syst. \& Contr. Letters, Vol.31, No.3, 1997.

[36] K. Zhou and P. P. Khargonekar, "Robust Stabilization on Linear Systems with Norm Bounded Time-Varying Uncertainty", Syst. \& Contr. Lett., Vol.10, No.1, pp.17-20, 1988.

[37] K. Zhou, “Essentials of Robust Control”, Prentice Hall Inc., New Jersey, USA, 1998. 


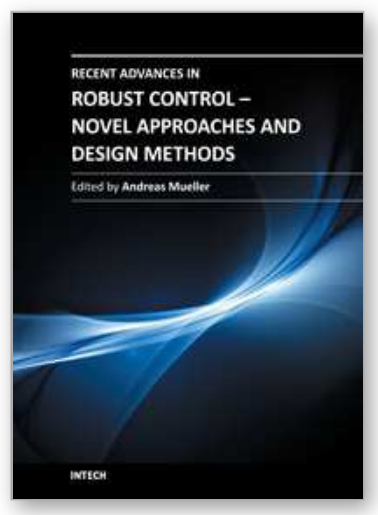

\author{
Recent Advances in Robust Control - Novel Approaches and \\ Design Methods \\ Edited by Dr. Andreas Mueller
}

ISBN 978-953-307-339-2

Hard cover, 462 pages

Publisher InTech

Published online 07, November, 2011

Published in print edition November, 2011

Robust control has been a topic of active research in the last three decades culminating in $\mathrm{H}$ 2/H_linfty and Imu design methods followed by research on parametric robustness, initially motivated by Kharitonov's theorem, the extension to non-linear time delay systems, and other more recent methods. The two volumes of Recent Advances in Robust Control give a selective overview of recent theoretical developments and present selected application examples. The volumes comprise 39 contributions covering various theoretical aspects as well as different application areas. The first volume covers selected problems in the theory of robust control and its application to robotic and electromechanical systems. The second volume is dedicated to special topics in robust control and problem specific solutions. Recent Advances in Robust Control will be a valuable reference for those interested in the recent theoretical advances and for researchers working in the broad field of robotics and mechatronics.

\title{
How to reference
}

In order to correctly reference this scholarly work, feel free to copy and paste the following:

Hidetoshi Oya and Kojiro Hagino (2011). Synthesis of Variable Gain Robust Controllers for a Class of Uncertain Dynamical Systems, Recent Advances in Robust Control - Novel Approaches and Design Methods, Dr. Andreas Mueller (Ed.), ISBN: 978-953-307-339-2, InTech, Available from:

http://www.intechopen.com/books/recent-advances-in-robust-control-novel-approaches-and-designmethods/synthesis-of-variable-gain-robust-controllers-for-a-class-of-uncertain-dynamical-systems

\section{INTECH}

open science | open minds

\section{InTech Europe}

University Campus STeP Ri

Slavka Krautzeka 83/A

51000 Rijeka, Croatia

Phone: +385 (51) 770447

Fax: +385 (51) 686166

www.intechopen.com

\section{InTech China}

Unit 405, Office Block, Hotel Equatorial Shanghai

No.65, Yan An Road (West), Shanghai, 200040, China

中国上海市延安西路65号上海国际贵都大饭店办公楼 405 单元

Phone: +86-21-62489820

Fax: $+86-21-62489821$ 
(C) 2011 The Author(s). Licensee IntechOpen. This is an open access article distributed under the terms of the Creative Commons Attribution 3.0 License, which permits unrestricted use, distribution, and reproduction in any medium, provided the original work is properly cited. 